\title{
BLACK HOLES WITHOUT SPACELIKE SINGULARITIES
}

\author{
MIHALIS DAFERMOS
}

\begin{abstract}
It is shown that for small, spherically symmetric perturbations of asymptotically flat two-ended Reissner-Nordström data for the EinsteinMaxwell-real scalar field system, the boundary of the dynamic spacetime which evolves is globally represented by a bifurcate null hypersurface across which the metric extends continuously. Under additional assumptions, it is shown that the Hawking mass blows up identically along this bifurcate null hypersurface, and thus the metric cannot be extended twice differentiably, in fact, cannot be extended in a weaker sense characterized at the level of the Christoffel symbols. The proof combines estimates obtained in previous work with an elementary Cauchy stability argument. There are no restrictions on the size of the support of the scalar field, and the result applies to both the future and past boundary of spacetime. In particular, it follows that for an open set in the moduli space of solutions around Reissner-Nordström, there is no spacelike component of either the future or the past singularity.
\end{abstract}

\section{INTRODUCTION}

It is well known that for the Kerr and Reissner-Nordström families of spacetimes, then unless the rotation or charge parameter vanishes, the maximal globally hyperbolic region $(\mathcal{M}, g)$ determined (as a solution to the Cauchy problem for the Einstein or Einstein-Maxwell system, respectively) by initial data is smoothly extendible to a larger spacetime $(\widetilde{\mathcal{M}}, \widetilde{g})$ so that all future incomplete causal geodesics $\gamma$ in $\mathcal{M}$ pass into the extension $\widetilde{\mathcal{M}} \backslash \mathcal{M}$. The boundary of $\mathcal{M}$ in $\widetilde{\mathcal{M}}$ defines what is known as a Cauchy horizon (denoted $\mathcal{C H}^{+}$) and is in fact a bifurcate null hypersurface. In the case of Reissner-Nordström, this situation can be illustrated formally in the well-known Penrose diagrammatic notation:

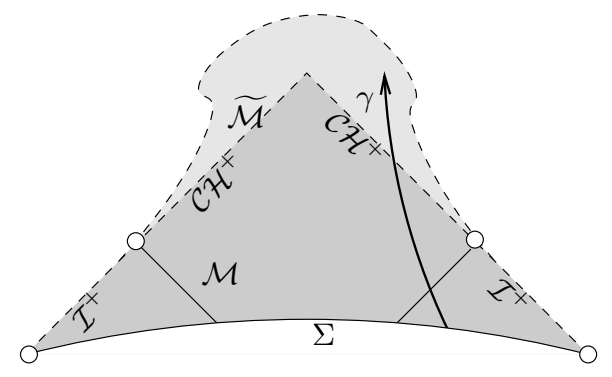

The above situation may be considered to be undesirable from the physical or epistemological point of view as it means that the fate of classical observers is not determined by what (from local considerations) would appear to be an impeccable initial hypersurface $\Sigma$. The question will not easily go away in view of a celebrated

University of Cambridge, Department of Pure Mathematics and Mathematical Statistics, Wilberforce Road, Cambridge CB3 0WB United Kingdom. 
result of Penrose 39] which implies in particular that if one perturbs the initial data on $\Sigma$, there will still always exist observers who, like $\gamma$ above, have incomplete worldlines. Thus, addressing the fate of such observers who live only for a finite time is an unavoidable issue for the theory.

From the above point of view, the situation in the celebrated Schwarzschild solution (thought of as a special case of the Reissner-Nordström/Kerr family corresponding to vanishing charge/rotation) can be considered to be preferable. Recall the Penrose diagramme:

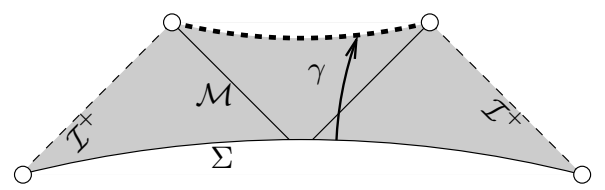

Here the curvature blows up along all incomplete inextendible causal geodesics $\gamma$ as the supremum of affine time is approached, and, in fact, one cannot extend nontrivially $\mathcal{M}$ to an $(\widetilde{\mathcal{M}}, \widetilde{g})$ such that $\widetilde{g}$ is a $C^{0}$ metric. This means that those macroscopic observers who live only for finite time do not just encounter infinite curvature but are pulled apart by an infinite tidal deformation. Though perhaps not so pleasant for those observers, this situation gives the classical theory an attractive sense of "closure". Macroscopic observers either live forever or are destroyed (as classically well-defined objects); no one is left unaccounted for!

1.1. Strong cosmic censorship. Since the Schwarzschild metrics constitute a one-parameter subfamily of Reissner-Nordström or Kerr, then in the class of explicit stationary solutions, it is the (undesirable) Reissner-Nordström/Kerr behaviour that would appear to be generic. It turns out, however, that the ReissnerNordström Cauchy horizon $\mathcal{C H}^{+}$is a surface of infinite blueshift, and an old heuristic argument due to Simpson and Penrose [43] suggested that this would cause it to be unstable to dynamic perturbations of the metric, which would be amplified by a blueshift effect. This motivated the so-called strong] cosmic censorship conjecture:

Conjecture (Strong cosmic censorship). For generic asymptotically flat initial data for "reasonable" Einstein-matter systems, the maximal Cauchy development is future inextendible as a suitably regular Lorentzian manifold.

The word "reasonable" above is meant to exclude in particular matter models for which the analogue of the above is false even when one does not couple to gravity?

\footnotetext{
${ }^{1}$ The word "strong" here is to differentiate between the so-called "weak" cosmic censorship, also due to Penrose, which states that for generic asymptotically flat data, future null infinity is complete (see 13 for a general formulation, and [12] for the seminal proof of this conjecture for the Einstein-scalar field model under spherical symmetry). This conjecture has the loose interpretation that inextendible, incomplete geodesics must enter black hole regions. In the formulations given, neither conjecture implies the other, but the Reissner-Nordström/Kerr behaviour, were it generic, would violate strong cosmic censorship, but not weak. The traditional epithets reflect precisely this relation.

${ }^{2}$ Here again, one must be careful as to which notion of maximal development (i.e. based on which regularity class) one considers. For the most classical of all matter models, the case of perfect fluids, for instance, the analogue of the above conjecture is false for the smooth maximal development (in view of the well known formation of shocks), but may be true for a notion of maximal development based on a wider class of solutions admitting shocks and their interactions. In the $1+1$ dimensional case, such a class can be built based on the space $B V$, at least for sufficiently small solutions. See the discussion in Kommemi 32 .
} 
In particular, the conjecture is meant to apply for the vacuum equations, where no matter is present. We shall comment on various formulations of 'suitably regular' further down.

According to the above conjecture, the Schwarzschild inextendibility property, though special in the class of explicit, stationary solutions, would turn out to be generic after all in the class of dynamical solutions. In particular, for this conjecture to be true, the internal structure of the black hole region of the ReissnerNordström/Kerr family would have to be unstable to perturbation of initial data.

Since at the time of the formulation of the above conjecture, Schwarzschild was the main example exhibiting the phenomenon postulated therein as generic, it naturally served as a basic model for the expected picture. In particular, it was expected that generically, the singular boundary of spacetime should be spacelike, meaning that typically, observers "falling into the singularity" eventually become causally independent, and, moreover, the singularity should be strong, meaning that the volume element (with respect to a suitable foliation) vanishes and macroscopic observers falling into the singularity are "crushed" (cf. 45]). Locally, it is well known that these properties are similar to the "big bang" type singularities of model cosmological spacetimes like FRW or the Bianchi class. (Indeed, the black hole interior of Schwarzschild may be considered a homogeneous "cosmological" solution with spatial topology $\mathbb{R} \times \mathbb{S}^{2}$ or quotients thereof.) Motivated primarily by the cosmological case, a much cited approach to uncover the form of the "generic singularity" attempted to infer (via a series of approximations) the existence of an infinite dimensional family of spacetimes of prescribed singular behaviour with free functions, naively identifying the latter with the degrees of freedom of initial data [3]. This approach was extremely influential in shaping a 'standard' picture of the interiors of generic black holes, represented by the Schwarzschild Penrose diagramme, but where the singularity exhibits behaviour as prescribed in 3 . This is sometimes known as the BKL scenario.

1.2. The mass inflation scenario and weak null singularities. This neat 'standard picture' of spacetime singularities fell apart (at least as a universal prescription) thanks to the careful study of what turned out to be a very fruitful class of toy models, namely spherically symmetric models of collapse in the presence of charge. These are the simplest models which on the one hand allow for the formation of Cauchy horizons (i.e. they admit Reissner-Nordström as a particular solution), while on the other hand, provide a dynamic degree of freedom on which the blue-shift mechanism can operate and affect a backreaction on the metric.

1.2.1. Null dust models. There is in fact a hierarchy of progressively more complicated spherically symmetric charged models one can consider. By far the simplest model quantifying backreaction is that of a self-gravitating ingoing null dust. Here the spacetime can be described by an explicit solution, the Reissner-NordströmVaidya metric. This was studied already in 1981 by Hiscock [27. These solutions admit a null boundary across which the spacetime is extendible continuously. All curvature scalars remain bounded on the boundary, suggesting at first instance that the spacetime is regular there, just as for the Reissner-Nordström Cauchy horizon; more careful analysis quickly reveals that various components blow up with respect to a freely falling frame. Thus, continuous spacetime extensions beyond the boundary fail to have $C^{2}$ metric, and the boundary can be viewed as a null singularity, 
whose singular nature is however not manifest in curvature scalars. In the language of [23, it is a "whimper".

The above model can easily be criticised in that it does not allow for any sort of backscattering of radiation. This fact, coupled perhaps with the general expectation that "whimper" singularities are unstable 23, led to the null geometry of the singularity not receiving the attention it deserved. In seminal work, Poisson and Israel 41, 40] "irradiated" the above spacetime with a second "outgoing" null dust, starting at a retarded time subsequent to the event horizon. The future evolution can no longer be represented as an explicit solution, but Poisson and Israel were nonetheless able to deduce that the Hawking mass would diverge on the null boundary as soon as one entered the domain of influence of the second null dust-hence "mass inflation". The divergence of the Hawking mass implied in particular that the Kretschmann scalar diverged-thus, the null boundary turned into a stronger "curvature singularity" (in the language of [23]).

It turned out, however, that the most interesting aspect of the singularity of Poisson and Israel [41, 40, was not that it was "stronger" than that of Hiscock [27, but rather, as shown by Ori 37, that it too was "weak", now from the point of Tipler's classification [45. Specifically, Ori 37 studied a slightly more tractable model where the ingoing null dust was replaced by a shell, and in the context of this model, showed that the backreaction would not be sufficient to immediately contract the area of the symmetry spheres to 0 , in fact, the metric would remain well behaved on the null boundary and could continue beyond. This implies in particular that macroscopic objects might not be "destroyed" by the singularity.

In retrospect, as we shall see, the proper characterization of the singularity in all of the above models 27, 41, 37, is not at the level of blow up of the curvature but at the level of the non-square integrability of the Christoffel symbols. This notion is perhaps less familiar than the traditional classifications of singularity [23, 45], but more relevant to pde properties governing the dynamics of the Einstein equations as well as the equations of continuum matter, for which the pointwise blow-up of curvature per se is of no particular significance 31. From this point of view, all above examples are in fact at the same level of singularity, which, though fully deserving of the appelation "weak" in view of the properties discovered by Ori [37, is at the same time sufficiently strong so as to be "terminal". We shall return to this issue after discussing the results for Einstein-Maxwell-real scalar field model in Section 1.2.4 See in particular footnote 4

1.2.2. The Einstein-Maxwell-real scalar field model. While better than the Vaidya model of [27, the above models [41, 37] are still open to criticism. Backscattering is put in by hand, and only occurs strictly after horizon formation; i.e. the above spacetimes coincide with the model of 27 in a small neighborhood of $i^{+}$. Moreover, the rate of decay of radiation before this artificial backscattering occurs is also put in by hand.

It was clear (see the discussion in [29]) that a more realistic model was necessary. In this regard, a natural candidate was the Einstein-Maxwell-real scalar field model. Here the Einstein equations are coupled to the Maxwell and wave equations, but the scalar field is uncharged, thus the latter two interact only through the gravitational coupling (see Section 2). 
The above model admits true wave-like behaviour and allows for the decay rate of radiation on the horizon to arise dynamically. It is a generalisation of the spherically symmetric Einstein-scalar field model whose mathematical study was initiated by Christodoulou leading to his seminal proofs of both weak and strong cosmic censorship [12. In contrast to Christodoulou's model, however, the Einstein-Maxwellreal scalar field equations have a peculiar feature: In view of the absence of charged matter, then for the Maxwell tensor to be non trivial, a complete spacelike hypersurface must have two asymptotically flat ends, just like in maximally extended Schwarzschild or Reissner-Nordström. We will return to this global feature in Section 1.3 as this will be central to considerations of the present paper. These global features per se do not however directly relate to the question of existence of weak null singularities arising from the "point" $i^{+}$of the Penrose diagramme, which can be addressed restricting to an arbitrary small neighbourhood of $i^{+}$.

1.2.3. The numerical and heuristic studies. The above model was first approached numerically, starting with a study by Gnedin and Gnedin [24. Though in retrospect, the numerics of 24 are best viewed as inconclusive (see the discussion of [4, 29]), the authors themselves interpreted their results as suggesting that the entire singular boundary is spacelike, casting doubt on the mass-inflation/weak null singularity scenario. The heuristic study of the scalar field model by Bonnano et al [4, on the other hand, gave arguments in favour of the scenario. This was followed finally by more careful numerical work by Brady-Smith [7, starting from data on the horizon, and later Burko [9] starting from characteristic data on a cone terminating on null infinity; the results of these later numerics supported the picture proposed by Poisson-Israel and Ori of a non-empty piece of null boundary emanating from $i^{+}$on which the mass blows up but across which the metric is continuously extendible.

1.2.4. The proof of the mass-inflation/weak null singularities scenario. The massinflation scenario and the associated weak null singularities were finally mathematically proven in [15, 17, to indeed occur in the Einstein-Maxwell-real scalar field model.

The first result concerns the persistence of a piece of null boundary of spacetime:

Theorem 1 (Cauchy horizon stability [17). For arbitrary asymptotically flat spherically symmetric data for the Einstein-Maxwell-real scalar field system for which the scalar field decays suitably at spatial infinity $i^{0}$, then if the charge is non-vanishing and the event horizon $\mathcal{H}^{+}$is asymptotically subextremal, it follows that the Penrose diagramme contains a subset which is as below

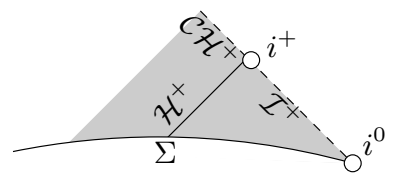

where $\mathrm{CH}^{+}$is a non-empty piece of null boundary. Moreover, the spacetime can be continued beyond $\mathrm{CH}^{+}$to a strictly larger manifold with $\mathrm{C}^{0}$ Lorentzian metric, to which the scalar field also extends continuously.

The $C^{0}$ extendibility statement captures the "weakness" of the singularity discussed by Ori in the context of [37. The above thus suggests that if by "sufficiently 
regular' in the formulation of strong cosmic censorship, one only requires that the metric extend $C^{0}$ (see 13]), then the conjecture is false.

The proof of the above mathematical theorem appeals in particular to the fact that Price's law tails hold as an upper bound for the behaviour of the scalar field on the horizon $\mathcal{H}^{+}$, a statement which in turn was proven in joint work with Rodnianski 20. See Theorem 5 of Section 7 . If such a polynomial decay rate is also assumed as a lower bound (cf. the numerical work [25] and Conjecture 2 in Section 8 below), then one obtains that the null boundary piece $\mathcal{C H}^{+}$is in fact singular:

Theorem 2 (Mass inflation [17). If a suitable lower bound on the decay rate of the scalar field on the event horizon $\mathcal{H}^{+}$is assumed, then the non-empty piece of null boundary $\mathrm{CH}^{+}$of Theorem 1 is in fact a weak null singularity on which the Hawking mass blows up identically. In particular, the metric cannot be continued beyond $\mathrm{CH}^{+}$as a $C^{2}$ metric. The scalar field cannot be extended beyond $\mathrm{CH}^{+}$as a $H_{\text {loc }}^{1}$ function.

We have stated above the $C^{2}$ inextendibility result for the metric because it is the easiest to give a self-contained proof in view of the fact that curvature is a geometric invariant. One expects to be able to make a much stronger statement, however, namely that the metric can in fact not be continued beyond $\mathrm{CH}^{+}$as a continuous metric with Christoffel symbols in $L_{\text {loc }}^{2} 3$ This notion of inextendibility, though not sufficient to show that macroscopic observers are torn apart, ensures that the boundary of spacetime is singular enough so that one cannot continue as weak solutions to a suitable Einstein-matter system. In this sense, it is sufficient to ensure a version of determinism. The above results suggest that "inextendiblity as a Lorentzian manifold with continuous metric and with Christoffel symbols in $L_{\text {loc }}^{2}$ " may be the correct formulation of "inextendible as a suitably regular Lorentzian metric" in the statement of strong cosmic censorship. This formulation is due to Christodoulou. See the discussion in the introduction of [14, 4

We remark finally that there is an additional level in the hierarchy of spherically symmetric models which one can consider-namely the Einstein-Maxwell-complex scalar field system. In this model, the scalar field carries charge which can source the Maxwell field, and thus charged black holes can form in collapse from data which are topologically Euclidean and contain no horizons. This model has been studied numerically in 28] while results similar to Theorem 1 (conditional, however, on an analogue of Theorem [5] have been obtained in upcoming work of Kommemi [34.

1.3. Global, bifurcate weak null singularities. As we have already discussed in Section 1.2.2, in contrast to the model of 28, 32, 34, just referred to, the EinsteinMaxwell-real scalar field system is such that for the Maxwell tensor to be nontrivial, complete initial data necessarily will have two asymptotically flat ends. The theorems of the previous section only probed the structure of the boundary of

\footnotetext{
${ }^{3}$ In [17, it is shown that the Christoffel symbols fail to be in $L_{\text {loc }}^{2}$ for a particular extension with continuous metric.

${ }^{4}$ We note that these inextendibility remarks apply equally to the models of Section 1.2 .1 In particular, from this point of view, the singularity in the model studied by Hiscock [27] is not really less singular than that of Poisson-Israel 41, when both are examined primarily from the perspective of the blow-up of their Christoffel symbols. In this regard, see also the discussion in [26].
} 
spacetime in a neighbourhood of $i^{+}$. What about the remaining boundary? This will be the subject of the present paper.

A preliminary result, using the fact that the matter model is, in the language of 32, "strongly tame", implies that, if the initial data hypersurface $\Sigma$ is moreover assumed to be "future admissible" (see Section 4), this boundary in general is as below:

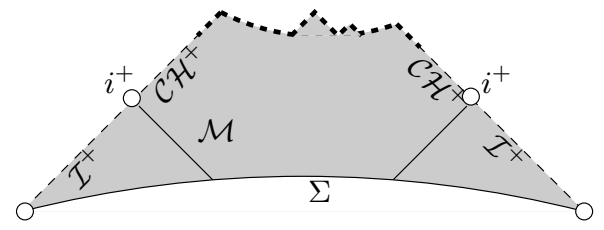

where in addition to the null boundary components $\mathcal{C H}^{+}$emanating from $i^{+}$, on which $r$ is bounded below (at this level of generality, these components are possibly empty, but are indeed non-empty if Theorem 1 applies), there is an (again, possibly empty!) achronal boundary on which $r$ extends continuously to 0 , depicted above as the thicker-shaded dotted line. See Proposition 4.1 for a precise statement. Note that the boundary decomposition implicit in the above diagramme already represents a non-trivial statement about possible singularity formation, as it excludes in particular "first" singularities corresponding to TIPs with compact intersection with $\Sigma$ and whose spherically symmetric spheres do not contract in the limit to zero area. In view of the topology of the initial data and the monotonicity properties inherent in the Einstein equations, it follows that $J^{-}\left(\mathcal{I}^{+}\right)$has (as depicted) a non-empty complement and $\mathcal{I}^{+}$is complete. Thus, in particular, the above result contains the statement of "weak" cosmic censorship 5

In this short paper, it is shown that when one restricts to suitably small perturbations of sub-extremal Reissner-Nordström, the $r=0$ boundary is in fact empty, and the two null components join to form a bifurcate null boundary across which the spacetime is globally extendible as a manifold with $C^{0}$ Lorentzian metric (to which the scalar field also extends continuously)! Moreover, if the assumption of Theorem 2 holds on both components of the event horizon $\mathcal{H}^{+}$, then the Hawking mass blows up identically on this bifurcate null boundary and the spacetime is inextendible as a Lorentzian manifold with $C^{2}$ metric, the scalar field is intextendible as an $H_{\text {loc }}^{1}$ function, and in fact, one can make a statement about the blow up of the $L^{2}$ norm of the Christoffel symbols. Specifically:

Theorem 3. Let $(\mathcal{M}, g, \phi, F)$ be the maximal development of sufficiently small spherically symmetric perturbations of asymptotically flat two-ended data corresponding to subextremal Reissner-Nordström with parameters $0<Q_{R N}<M_{R N}$, under the evolution of the Einstein-Maxwell-real scalar field system.

\footnotetext{
${ }^{5}$ We emphasise, however, that this is a very soft result in comparison with Christodoulou's seminal proof 12 of weak cosmic censorship in the case of one end, where the main obstacle is null boundary components emanating from the centre of symmetry. In the two-end case, there is no such centre and thus, a fortiori, no null boundary components of the above type.
} 
Then there exists a later Cauchy surface $\Sigma_{+}$which is future-admissible and such that to the future of $\Sigma_{+}$, the Penrose diagramme of $(\mathcal{M}, g)$ is given by:

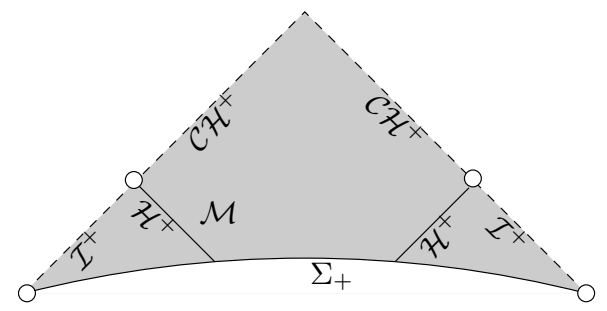

Similarly, there exists also an earlier Cauchy surface $\Sigma_{-}$which is past-admissible, such that to the past of $\Sigma_{-}$, the Penrose diagramme of $(\mathcal{M}, g)$ is given by a time reversed depiction of the above, with boundary components $\mathcal{I}^{-}, \mathrm{CH}^{-}$, etc.

The global bound

$$
r \geq M_{R N}-\sqrt{M_{R N}^{2}-Q_{R N}^{2}}-\epsilon
$$

holds for the area-radius $r$ of the spherically symmetric spheres, where $\epsilon \rightarrow 0$ as the 'size' of the perturbation tends to 0. Moreover, the metric extends continuously beyond $\mathcal{C H}^{+}$to a strictly larger Lorentzian manifold $(\widetilde{\mathcal{M}}, \widetilde{g})$, making $\mathcal{C H}^{+}$a bifurcate null hypersurface in $\widetilde{\mathcal{M}}$. The scalar field $\phi$ extends to a continuous function on $\widetilde{\mathcal{M}}$. All future-incomplete causal geodesics in $\mathcal{M}$ extend to enter $\widetilde{\mathcal{M}}$.

Finally, if $\phi$ satisfies the assumption of Theorem 回 on both components of the horizon $\mathcal{H}^{+}$, then the Hawking mass extends "continuously" to $\infty$ on all of $\mathcal{C H}^{+}$. In particular, $(\mathcal{M}, g)$ is future inextendible as a spacetime with $C^{2}$ Lorentzian metric, and continuous extensions $\phi$ as in the previous paragraph fail to be $H_{\mathrm{loc}}^{1}$ in a neighborhood of every point of $\partial \mathcal{M} \subset \widetilde{\mathcal{M}}$.

Exactly analogous statements hold for $\mathcal{H}^{-}, \mathrm{CH}^{-}$.

In fact, one can hope to show that, under the assumption of Theorem 2, given an extension $(\widetilde{\mathcal{M}}, \widetilde{g})$ with $C^{0}$ metric, the Christoffel symbols fail to be $C^{0,1}$ everywhere on $\mathcal{C H}^{+}$, and, wherever $\left.\phi\right|_{\mathcal{C H}^{+}}$is not locally constant, the Christoffel symbols in fact fail to be locally $L^{2}$. In particular, the latter holds in a neighbourhood of $i_{A, B}^{+}$. As in [17, we will here, however, only show these statements for a particular $C^{0}$ extension and not address the geometric issues in generalising this for all such extensions.

If one only considered, say, future evolution, the proof of the stability part of the above theorem would be much easier (but much less interesting) if one moreover restricted to data which is supported strictly between the two components of the future event horizon $\mathcal{H}^{+}$, as such solutions would coincide with Reissner-Nordström in a neighbourhood of $i^{+}$. Evolving to the past, however, this property would be lost. We have stated our result as a statement about both future and past evolution to emphasise that we are not dealing with that essentially uninteresting case. In fact, our smallness condition does not require that $\phi$ be initially of compact support.

The analogy of the Reissner-Nordström and Kerr families suggests that the situation for solutions of the vacuum equations (with no symmetry assumed!) in a neighbourhood of the Kerr family may be similar. This is further supported by a perturbative analysis by Ori [38. One may thus conjecture: 
Conjecture 1 (A. Ori). Let $(\mathcal{M}, g)$ be the maximal vacuum Cauchy development of sufficiently small perturbations of asymptotically flat two-ended Kerr data corresponding to parameters $0<|a|<M$. Then there exist both a future and past extension $(\widetilde{\mathcal{M}}, \widetilde{g})$ of $\mathcal{M}$ with $C^{0}$ metric $\widetilde{g}$ such that $\partial \mathcal{M}$ is a bifurcate null cone in $\widetilde{\mathcal{M}}$ and all future (past) incomplete geodesics in $\gamma$ pass into $\widetilde{\mathcal{M}} \backslash \mathcal{M}$.

Moreover, for generic such perturbations, any $C^{0}$ extension $\widetilde{\mathcal{M}}$ will fail to have $L^{2}$ Christoffel symbols in a neighbourhood of any point of $\partial \mathcal{M}$.

Thus, according to the above conjecture, not only should black holes generically have part6 of their singular boundary null, but a small neighbourhood (in the moduli space of vacuum metrics) of the Kerr family would, in both future and past, generically be entirely free of spacelike singularities.

We end this introduction with a final remark: Until a few years ago, "large data" problems for the Einstein vacuum equations without symmetry seemed mathematically intractable. This view changed completely with the seminal work of Christodoulou [14] on black hole formation in vacuum, which demonstrated how largeness can be "tolerated" in the analysis as long as it appears in a controlled way and is "coupled" with a corresponding "smallness". In broad terms, this is in fact quite reminiscent of structure that plays a role in the analysis here. An even more directly related link is provided by the recent Luk-Rodnianski theory [35, 36] of interacting impulsive gravitational waves, reviewed briefly in Section 14 of this paper, which illuminates a new hiearchy in the vacuum equations which is almost certainly key to understanding solutions in the presence of null singularities. These works, coupled with progress on the stability of black hole exterior regions (surveyed in 22., cf. the role of Theorem [5), give hope that Conjecture 1, and its "cosmological twin" Conjecture 3-but also, quite ominously, Conjecture 5-both stated in Section [15, will soon be amenable to mathematical analysis.

\section{The Einstein-MAXWelL-(REAL)-SCALAR FIELD MOdeL}

The Einstein-Maxwell-(real)-scalar field system describes the interaction of a gravitational field, an electromagnetic field, and an uncharged scalar field. The latter two thus interact solely via their coupling to gravity. The equations are given by

$$
\begin{gathered}
R_{\mu \nu}-\frac{1}{2} g_{\mu \nu} R=8 \pi T_{\mu \nu}, \\
\nabla^{\mu} F_{\mu \nu}=0, \quad \nabla_{[\lambda} F_{\mu \nu]}=0, \\
\square_{g} \phi=0,
\end{gathered}
$$

\footnotetext{
${ }^{6}$ We emphasise explicitly that the implication that part of the singular boundary be null, which is already suggested by Theorem [1] and 2] should apply to generic black holes forming in collapse from one asymptotically flat end, in view of the fact that it is believed that these always approach Kerr in a neighbourhood of $i^{+}$in the domain of outer communications. The above conjecture cannot however address the global structure of the boundary of such spacetimes as these are globally far from the Kerr family. We note, for instance, that the numerical work of [28 in the context of the spherically symmetric charged scalar field model suggests, in addition to a null component, also a non-empty spacelike portion of the singularity in the case of collapse of data with one end. See also Conjecture 1.11 of $[32$ and subsequent comments therein.
} 


$$
T_{\mu \nu}=\partial_{\mu} \phi \partial_{\nu} \phi-\frac{1}{2} g_{\mu \nu} \nabla^{\alpha} \phi \nabla_{\alpha} \phi+\frac{1}{4 \pi} F_{\mu}^{\alpha} F_{\alpha \nu}-\frac{1}{16 \pi} g_{\mu \nu} F^{\alpha \beta} F_{\alpha \beta} .
$$

Let us recall the version of the foundational well posedness theorem for general relativity, which applies for the above model:

Theorem ([11]). Let $\left(\Sigma, \bar{g}, K, \bar{F}_{a b}, \bar{F}_{0 b}, \phi_{0}, \phi_{1}\right)$ be a smooth Einstein-Maxwell-scalar field data set. Then there exists a unique non-empty maximal smooth Cauchy development $\left(\mathcal{M}, g, F_{\mu \nu}, \phi\right)$ for the equations (11)-(4).

The motivation for the above system has been discussed already in Section 1.2.2 and at length in [15, 17. It is perhaps the simplest system admitting ReissnerNordström as an explicit solution and allowing at the same time for non-trivial wave dynamics in spherical symmetry.

\section{THE SPHERICALly SYMMETRIC REDUCTION}

In the case of spherically symmetric data, then the maximal Cauchy development is spherically symmetric and the Einstein equations reduce to a $1+1$ dimensional system.

For the purpose of this discussion, by spherically symmetric data set we assume that $(\Sigma, \bar{g})$ is a warped product $\mathbb{R} \times_{r} \mathbb{S}^{2}$, with metric $d x^{2}+r^{2}(x) d \sigma_{\mathbb{S}}^{2}$ (admitting the obvious $\mathrm{SO}(3)$-action), $K_{a b}$ is an $\mathrm{SO}(3)$-invariant symmetric 2 -tensor on $\mathbb{R} \times{ }_{r} \mathbb{S}^{2}, \phi_{0}$, and $\phi_{1}$ are functions on $\mathbb{R}$, and $\bar{F}_{a b}, \bar{F}_{0 b}$ are an $\mathrm{SO}(3)$ invariant 2 -form and vector field, respectively, on $\mathbb{R} \times_{r} \mathbb{S}^{2}$, together satisfying the Einstein-Maxwell-real scalar field constraint equations. These equations can be found, for instance, in [20].

Proposition 3.1. Let $(\mathcal{M}, g, F, \phi)$ be the maximal Cauchy development of smooth spherically symmetric data as described above. Then $\mathcal{M}$ is spherically symmetric and is moreover of the form of a warped product $(\mathcal{M}, g)=\mathcal{Q} \times_{r} \mathbb{S}^{2}$, where $\mathcal{Q}$ admits a global null coordinate system $(u, v)$, and the metric takes the form

$$
-\Omega^{2}(u, v) d u d v+r^{2}(u, v) \sigma_{\mathbb{S}^{2}} .
$$

The Maxwell field takes the form

$$
F=\frac{\Omega^{2} Q_{e}}{2 r^{2}} d u \wedge d v+Q_{m} \sin \theta d \theta \wedge d \varphi
$$

for real constants $Q_{e}, Q_{m}$, and $\phi$ descends to a function $\phi: \mathcal{Q} \rightarrow \mathbb{R}$. Defining $Q=\sqrt{Q_{e}^{2}+Q_{m}^{2}}$, the full content of the equations is thus given by

$$
\begin{gathered}
\partial_{u} \partial_{v} r=-\frac{\Omega^{2}}{4 r}-\frac{1}{r} \partial_{v} r \partial_{u} r+\frac{1}{4} \Omega^{2} r^{-3} Q^{2} \\
\partial_{u} \partial_{v} \log \Omega^{2}=-4 \pi \partial_{u} \phi \partial_{v} \phi+\frac{\Omega^{2}}{4 r^{2}}+\frac{1}{r^{2}} \partial_{v} r \partial_{u} r-\frac{\Omega^{2} Q^{2}}{2 r^{4}}, \\
\partial_{u}\left(r \partial_{v} \phi\right)=-\partial_{u} \phi \partial_{v} r \\
\partial_{u}\left(\Omega^{-2} \partial_{u} r\right)=-4 \pi r \Omega^{-2}\left(\partial_{u} \phi\right)^{2} \\
\partial_{v}\left(\Omega^{-2} \partial_{v} r\right)=-4 \pi r \Omega^{-2}\left(\partial_{v} \phi\right)^{2} .
\end{gathered}
$$


Let us note that we have a gauge freedom in defining null coordinates $\bar{u}=f(u)$, $\bar{v}=g(v)$, for general smooth functions $f, g$. We will normalise expedient null coordinate systems later on.

In "translating" intuition from the above system to the problem of vacuum collapse, the reader familiar with the formalism of [14] should note that $r \partial_{v} r$ corresponds to tr $\chi$, while it is useful to think of $r \partial_{v} \phi$ as an analogue of $\hat{\chi}$, even though in the spherically symmetric model, $r \partial_{v} \phi$ appears in the geometry (via the coupling) at the level of curvature.

\section{THE GENERAL FRAMEWORK}

We will consider spherically symmetric, asymptotically flat data $(\Sigma, \ldots)$ with two ends. Let us label the ends $A$ and $B$, and let us restrict our $(u, v)$ coordinate system such that asymptotically on $\Sigma, \partial_{v}$ points towards end $A$, and $\partial_{u}$ points towards $B$.

In order to obtain a "good" a priori characterization of the boundary of the maximal development, it is convenient to restrict to data such that

$$
\Sigma=\Sigma_{A} \cup \Sigma_{B}
$$

where $\Sigma_{A}$ satisfies $\partial_{u} r<0, \Sigma_{B}$ satisfies $\partial_{v} r<0$, and $\Sigma_{A}, \Sigma_{B}$ are connected. We shall call two-ended asymptotically flat data satisfying (10) future admissible. Let us note that asymptotic flatness requires that the end $A$ is "contained" in $\Sigma_{A}$ and disjoint from $\Sigma_{B}$, and similarly, the end $B$ is "contained" in $\Sigma_{B}$ and disjoint from $\Sigma_{A}$.

Note that Cauchy hypersurfaces in Schwarzschild or Reissner-Nordström are future admissible if they have no intersection with the closure of the white hole region in $\mathcal{Q}$.

The significance of the decomposition (10) is that by Raychaudhuri (8), (9) it follows that $\mathcal{Q} \cap J^{+}(\Sigma)=\left\{\partial_{u} r<0\right\} \cup\left\{\partial_{v} r<0\right\}$. It serves as a generalisation of Christodoulou's "no antitrapped surfaces" condition [13, appropriately reworked for the two-ended case. Note finally that the property (10) is stable to perturbation of data.

For future admissible data we have the following result which in fact follows from estimates shown in [17, but is best viewed as a special case of more general results of Kommemi 32 concerning the Einstein-Maxwell-complex scalar field system:

Proposition 4.1. Let $\left(\mathcal{M}=\mathcal{Q} \times_{r} \mathbb{S}^{2}, \Omega^{2}, \phi, Q_{e}, Q_{m}\right)$ be the maximal Cauchy development of data as above, with $\Sigma$ future admissible.

I. Then, in the notation of 32 , the Penrose diagramme of $(\mathcal{M}, g)$ to the future of $\Sigma$ is as below:

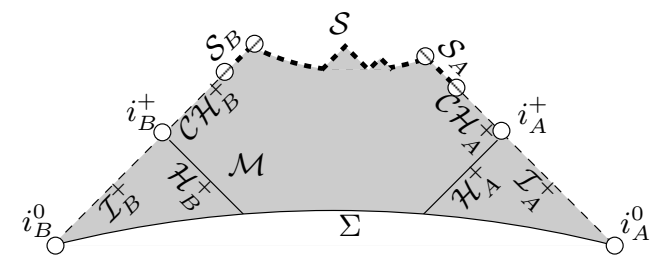

with the usual convention that some of the boundary components may be empty. 
II. Under the above assumptions, then $\mathcal{I}_{A, B}^{+}$is complete, the horizons $\mathcal{H}_{A, B}^{+}$are non-empty and a Penrose inequality $\sup _{\mathcal{H}_{A, B}^{+}} r \leq 2 M_{A, B}^{f}$ holds on each horizon, where $M_{A, B}^{f}$ is the infimum of the Bondi mass of $\mathcal{I}_{A, B}^{+}$, respectively.

III. Suppose $(\widetilde{\mathcal{M}}, \widetilde{g})$ is a $C^{2}$ future-extension of $(\mathcal{M}, g)$. Then, denoting by $\pi_{Q}$ : $\mathcal{M} \rightarrow \mathcal{Q}$ the natural projection, there exists a causal curve $\gamma$ passing into $\widetilde{\mathcal{M}} \backslash \mathcal{M}$ such that $\overline{\pi_{\mathcal{Q}}\left(\left.\gamma\right|_{\mathcal{M}}\right)} \cap\left(\mathcal{C H}_{A}^{+} \cup \mathcal{C H}_{B}^{+}\right) \neq \emptyset$.

We review very briefly some properties encoded in the above notation (see 32 . for details). The boundary segments $\mathcal{I}_{A, B}^{+}$have the property that $r$ extends "continuously" to $\infty$. We identify $\mathcal{I}_{A, B}^{+}$with "future null infinity". The notation $i_{A, B}^{+}$in fact already encodes the statement that "future null infinity is complete" (cf. Part II above). Monotonicity properties (cf. Section 5 below) allow for the definition of a nonnegative Bondi mass function on $\mathcal{I}_{A, B}^{+}$whose infimum defines the final Bondi mass $M_{A, B}^{f}$. The sets $\mathcal{C} \mathcal{H}_{A, B}^{+}$are half-open segments emanating from (but not including) $i_{A, B}^{+}$characterized by the property that 0 is not a limit point of $r$ on their interior, whereas $r$ extends continuously to 0 on $\mathcal{S}_{A} \cup \mathcal{S}_{B} \cup \mathcal{S}$.

Note that since by Part II, $J^{-}\left(\mathcal{I}^{+}\right)$has indeed a non-trivial complement, we have $\mathcal{C H}_{A}^{+} \cup \mathcal{C H}_{B}^{+} \cup \mathcal{S}_{A} \cup \mathcal{S}_{B} \cup \mathcal{S} \neq \emptyset$. Any 4 of the above 5 components, however, can be empty.

We emphasise that Proposition 4.1 is in fact a very general result for spherically symmetric Einstein-matter systems, holding for all matter-models which, in the terminology introduced by Kommemi [32, are "strongly tame". Another example of a strongly tame matter model is the Einstein-Vlasov system. See 21].

Note finally that a Cauchy hypersurface which is future admissible as we have defined it cannot be admissible to past evolution. In Reissner-Nordström or Schwarzschild, then Cauchy hypersurfaces are past-admissible iff they do not intersect the closure of the black hole region. On the other hand, by a Cauchy stability argument, one can show that given an arbitrary spherically symmetric asymptotically flat Cauchy hypersurface of Reissner-Nordström, then for suitably small perturbations of initial data, there will exist a future admissible Cauchy surface in the future, and a past-admissible Cauchy surface in the past which coincide for large $r$ with the original hypersurface. See Corollary 9.1 of Section 9 ,

\section{The trapped REgion, EVEnt horizons, AND OUtermost APPARENT HORIZONS}

We assume in this section that $\mathcal{Q}$ is given by Proposition 4.1. We will restrict consideration to $J^{+}(\Sigma)$, i.e. by $\mathcal{Q}$ below we shall actually mean $J^{+}(\Sigma) \cap \mathcal{Q}$. The notation $J^{-}\left(\mathcal{I}^{+}\right)$, etc., is understood in the usual sense i.e. as $J^{-}\left(\mathcal{I}^{+}\right) \cap \mathcal{Q}\left(\cap J^{+}(\Sigma)\right)$, where $J^{-}$denotes now the causal structure of the ambient $1+1$ dimensional Minkowski space defining the Penrose diagrammes. Let us note that for $p \in \mathcal{Q}$, then $J_{\mathcal{Q}}^{+}(p)=J_{\mathbb{R}^{1+1}}^{+}(p) \cap \mathcal{Q}$ so there is no danger of confusion. Closure on the other hand will be always with respect to the ambient topology of $\mathbb{R}^{1+1}$.

Let us define the trapped region:

$$
\mathcal{T}=\left\{(u, v) \in \mathcal{Q}: \partial_{u} r<0, \partial_{v} r<0\right\} .
$$

Our definition of admissibility (10) implies that $\mathcal{T} \cap \Sigma \neq \emptyset$, in particular,

$$
\mathcal{T} \neq \emptyset \text {. }
$$


The Raychaudhuri equations (8), (9) then imply that $\partial_{v} r>0$ in $J^{-}\left(\mathcal{I}_{A}^{+}\right), \partial_{u} r>0$ in $J^{-}\left(\mathcal{I}_{B}^{+}\right)$. We recall the definition of $\mathcal{H}_{A}^{+}$as the future boundary of $J^{-}\left(\mathcal{I}_{A}^{+}\right)$in $\mathcal{Q}$, and similarly $\mathcal{H}_{B}^{+}$. It follows that $\partial_{v} r \geq 0$ on $\mathcal{H}_{A}^{+}$, while $\partial_{u} r \geq 0$ on $\mathcal{H}_{B}^{+}$.

By our definition of admissibility, we easily see that on $\overline{J^{-}\left(\mathcal{I}_{A}^{+}\right)} \cap \mathcal{Q}$, and thus, by Raychaudhuri (8) also on $J^{+}\left(\mathcal{H}_{A}^{+}\right)$, we must have $\partial_{u} r<0$, while similarly, on $\overline{J^{-}\left(\mathcal{I}_{B}^{+}\right)} \cap \mathcal{Q}$, and thus, by (9), also on $J^{+}\left(\mathcal{H}_{B}^{+}\right)$, we must have $\partial_{v} r<0$.

Let us finally note that in view of the above, we indeed have $\mathcal{H}_{A}^{+} \cap \mathcal{H}_{B}^{+}=\emptyset$, as has been depicted and

$$
\mathcal{T} \cap \overline{J^{-}\left(\mathcal{I}_{A, B}^{+}\right)}=\emptyset
$$

We define $\mathcal{A}_{A}^{\prime} \subset J^{+}\left(\mathcal{H}_{A}^{+}\right)$to be the set $(u, v) \in J^{+}\left(\mathcal{H}_{A}^{+}\right)$such that $\partial_{v} r(u, v)=$ 0 but $\partial_{v} r\left(u^{*}, v\right)>0$ for $u^{*}<u$. We call $\mathcal{A}_{A}^{\prime}$ the outermost apparent horizon corresponding to end $A$.

Our assumptions in fact imply the non-emptiness of $\mathcal{A}_{A}^{\prime}$. For this, we first notice that, in view of both (8), (9), we have $\mathcal{C H}_{B}^{+} \cap \overline{J^{+}\left(\mathcal{H}_{A}^{+}\right)} \subset \overline{\mathcal{T}}$. Similarly, we have

$$
\mathcal{S}_{A} \cup \mathcal{S}_{B} \cup \mathcal{S} \subset \overline{\mathcal{T}}
$$

(Indeed, if $r(u, v)<\inf _{x \in \Sigma} r(x)$, then $(u, v) \in \mathcal{T}$.) It follows that along every ingoing null ray from $\mathcal{H}_{A}^{+}$, one must encounter a unique point of $\mathcal{A}_{A}^{\prime}$.

We define $\mathcal{A}_{B}^{\prime}$ analogously.

We emphasise that $\mathcal{A}_{A, B}^{\prime}$ as defined can be seen to be achronal sets, but are not necessarily connected.

Finally, let us remark that the above notion of trapped region $\mathcal{T}$ is defined by restricting attention to surfaces of symmetry. For a general discussion of nonspherically symmetric trapped surfaces in spherically symmetric ambient spacetimes, see [42].

\section{The HaWking Mass}

Recall the important quantity

$$
m=\frac{r}{2}\left(1-|\nabla r|^{2}\right)=\frac{r}{2}\left(1+4 \Omega^{-2} \partial_{u} r \partial_{v} r\right)
$$

known as the Hawking mass.

We remark that the Hawking mass is at the level of first derivatives of the metric. We also note, however, the inequality

$$
R_{\mu \nu \alpha \beta} R^{\mu \nu \alpha \beta} \geq \frac{4}{r^{4}}\left(\frac{2 m}{r}\right)^{2}
$$

for the Kretschmann scalar.

The so-called renormalised Hawking mass

satisfies

$$
\varpi=m+\frac{Q^{2}}{2 r}
$$

$$
\begin{aligned}
& \partial_{u} \varpi=-8 \pi r^{2} \Omega^{-2} \partial_{v} r\left(\partial_{u} \phi\right)^{2}, \\
& \partial_{v} \varpi=-8 \pi r^{2} \Omega^{-2} \partial_{u} r\left(\partial_{v} \phi\right)^{2} .
\end{aligned}
$$

We see the monotonicity properties

$$
\partial_{u} \varpi \geq 0, \quad \partial_{v} \varpi \geq 0 \quad \text { in } \mathcal{T},
$$


while

$$
\partial_{u} \varpi \leq 0, \quad \partial_{v} \varpi \geq 0 \quad \text { in } \overline{J^{-}\left(\mathcal{I}_{A}^{+}\right)} \cup\left(J^{+}\left(\mathcal{H}_{A}^{+}\right) \cap J^{-}\left(\mathcal{A}_{A}^{\prime}\right)\right),
$$

and similarly,

$$
\partial_{v} \varpi \leq 0, \quad \partial_{u} \varpi \geq 0 \quad \text { in } \overline{J^{-}\left(\mathcal{I}_{B}^{+}\right)} \cup\left(J^{+}\left(\mathcal{H}_{B}^{+}\right) \cap J^{-}\left(\mathcal{A}_{B}^{\prime}\right)\right) .
$$

Since by (11) we have $0<r=2 m$ on $\mathcal{A}_{A, B}^{\prime}$, it follows that on $\mathcal{A}_{A, B}^{\prime}$ we have $\varpi \geq Q$ and $\varpi>0$.

Defining now the asymptotic renormalised Hawking mass $\varpi_{A, B}^{+}$on each of the two horizons $\mathcal{H}_{A, B}^{+}$by

$$
\varpi_{A, B}^{+}=\sup _{\mathcal{H}_{A, B}^{+}} \varpi
$$

we have by our monotonicity properties that

$$
0<\varpi_{A, B}^{+} \leq M_{A, B}^{f} \leq M_{A, B}^{A D M}<\infty .
$$

where $M_{A, B}^{f}, M_{A, B}^{A D M}$ denote the final Bondi and ADM mass associated to each end, as well as

$$
Q \leq \varpi_{A, B}^{+}
$$

Interestingly, (16) can be proven given only the solution in $\mathcal{H}^{+} \cup J^{-}\left(\mathcal{I}^{+}\right)$under the assumption that $r$ is bounded above on $\mathcal{H}^{+}$, i.e. without using $\mathcal{A}_{A, B}^{\prime} \neq \emptyset$. See 17 .

Let us note that (16) allows for the case

$$
Q=\varpi_{A, B}^{+} .
$$

This is the case of an asymptotically extremal black hole (from the perspective of the event horizon 7). As we shall see, Theorem 4 below will have to exclude (17) in its statement. It is an open question to understand the dynamics sufficiently so as to characterize the causal structure of the boundary in the case (17), even in a neighbourhood of $i_{A, B}^{+}$. See the remarks in 32 .

A much more general discussion, including some of the statements given here, is contained in Kommemi 33 .

\section{Persistence of the null boundary: $\mathcal{C H}_{A, B}^{+} \neq \emptyset$}

We now give a more precise version of the first theorem of [17] (Theorem 10 of the introduction) adapted to the current framework.

For this, let us first normalise the null coordinates $u$ and $v$. Given $\Sigma$ with metric $d x^{2}+r^{2}(x) d \sigma_{\mathbb{S}^{2}}$, where $x \rightarrow \infty$ corresponds to end $A, x \rightarrow-\infty$ corresponds to end $B$, we pick an arbitrary origin and normalise our null coordinates such that $\partial_{u} x=-1, \partial_{v} x=1$ on $\Sigma$. We note that our notion of asymptotic flatness then guarantees that these coordinates are Eddington-Finkelstein-like, in the sense that $\partial_{u} r$ is uniformly bounded above and away from 0 on $\mathcal{I}_{B}^{-}, \mathcal{I}_{A}^{+}$, and similarly $\partial_{v} r$ on $\mathcal{I}_{A}^{-}, \mathcal{I}_{B}^{+}$(in small neighborhoods of $i_{A, B}^{0}$ ).

\footnotetext{
${ }^{7}$ cf. the condition $Q=M_{A, B}^{f}$ discussed in 33
} 
Theorem 4 (17]). Under the assumptions of Proposition 4.1 and the above normalisation of the null coordinates, let us in addition require that initially on $\Sigma$,

$$
\left|\partial_{u}(r \phi)\right| \leq C r^{-2}, \quad\left|\partial_{v}(r \phi)\right| \leq C r^{-2},
$$

for some arbitrary constant $C$. Then if

$$
0<Q<\varpi_{A, B}^{+},
$$

then

$$
\mathcal{C H}_{A, B}^{+} \neq \emptyset \text {. }
$$

Moreover, there exist neighourhoods $\mathcal{U}_{A, B}$ of $i_{A, B}^{+}$such that $\mathcal{A}_{A, B}^{\prime} \cap \mathcal{U}_{A, B}$ is a connected achronal curve terminating at $i_{A, B}^{+}$, and such that $D^{+}\left(\mathcal{A}_{A, B}^{\prime}\right) \subset \mathcal{T}$.

The second statement above signifies that $\mathcal{A}_{A, B}^{\prime} \cap \mathcal{U}_{A, B}$ is what is sometimes known as a 'dynamical horizon', except that it may contain outgoing null pieces. Such null pieces will in fact be excluded under the assumptions of Theorem 6 in the next section. See 46 for various general results about $\mathcal{A}^{\prime}$ in spherically symmetric spacetimes.

Let us note that the proof of Theorem 4 required to first establish a version of "Price's law"-type decay for the scalar field along the event horizons $\mathcal{H}_{A, B}^{+}$. This was proven in joint work with Rodnianski 20. As this has independent interest, we give the statement relevant for data satisfying (18):

Theorem 5 (M.D.-Rodnianski 20]). Under the assumptions (18), (19) of Theorem 4, then for all $\epsilon>0$,

$$
|\phi|+\left|\partial_{v} \phi\right| \leq \tilde{C}_{\epsilon} v^{-2+\epsilon}, \quad|\phi|+\left|\partial_{u} \phi\right| \leq \tilde{C}_{\epsilon} u^{-2+\epsilon}
$$

on $\mathcal{H}_{A}^{+}, \mathcal{H}_{B}^{+}$respectively, for a $\tilde{C}_{\epsilon}$ depending only on $\epsilon, C, \varpi_{A, B}^{+}$and $Q$.

For data where $\phi$ is of compact support on $\Sigma$, the decay bounds in the statement of Theorem 5 on the right hand side of (21) can be improved to $\tilde{C}_{\epsilon} v^{-3+\epsilon}, \tilde{C}_{\epsilon} u^{-3+\epsilon}$, illuminating the familiar obstruction at power 3. It is well known, however, that general semilinear problems even on a fixed Minkowski background already lead to decay which is captured by inverse polynomial decay weaker than $t^{-3}$, even for compactly supported data. Thus, it is far preferable to state Theorem 5 in the above more general context of data satisfying only (18), where the statements are more robust.

Finally, let us remark that assumption (19) is a "teleological" assumption. Note, however, that in view of the monotonicity properties of Section 5 , it follows immediately that (19) is indeed satisfied if it is assumed for example that initially, $\varpi>Q$ on $\Sigma$. We can already state in particular:

Corollary 7.1. If our initial data as in Proposition 4.1 is assumed sufficiently close to the data of a given subextremal Reissner-Nordström solution, then (19) is indeed satisfied and the conclusions of the above theorem hold.

$$
\text { 8. } \mathcal{C H}_{A, B}^{+} \text {IS (GLOBAlly) Singular }
$$

Under an additional assumption on the behaviour of the scalar field on $\mathcal{H}_{A, B}^{+}$, it is shown that $\mathcal{C H}_{A, B}^{+}$in fact correspond to weak null singularities. These can be thought of as singular null hypersurfaces in a $C^{0}$ extension of the metric, singular 
in that the Kretschmann scalar blows up, in fact, so that the Christoffel symbols are singular in a sense to be discussed in Section 13 .

Specifically, the following result was proven in [17:

Theorem 6 (17]). Under the assumptions of Theorem 4 suppose we have in addition that

$$
\left|\partial_{v} \phi\right| \geq c v^{-6+\tau}, \quad\left|\partial_{u} \phi\right| \geq c u^{-6+\tau}
$$

on $\mathcal{H}_{A}^{+}, \mathcal{H}_{B}^{+}$, for all $v \geq V, u \geq U$ respectively, for some $U, V<\infty$, and some $c>0, \tau>0$. Then $\varpi$ extends "continuously" to $\infty$ on $\mathcal{C H}_{A, B}^{+}$in a neighbourhood of $i_{A, B}^{+}$.

Moreover, choosing $\mathcal{U}_{A, B}$ in Theorem 4 sufficiently small, then the curves $\mathcal{A}_{A, B}^{\prime} \cap$ $\mathcal{U}_{A, B}$ are in fact spacelike.

The reader should compare the required lower bounds (22) with the upper bounds (21) proven in Theorem 5. (In particular, (22) is indeed compatible with (21)!). We have in fact the following

Conjecture 2. For generic data as in Thereom 4 the assumptions of Theorem 6 hold.

Resolution of the above would completely close the book on the mass-inflation scenario in the context of Einstein-Maxwell-real scalar field model, as the blow-up part of the scenario would then be retrieved for generic data posed on a spacelike asymptotically flat Cauchy surface.

We proceed in the remainder of this section to strengthen slightly Theorem 6 to show that $\varpi$ extends "continuously" to $\infty$ on the entire $\mathcal{C H}_{A, B}^{+}$.

First, note that in view of the second statement of Theorem 4 , we have that there exists an open neighbourhood $\mathcal{U}_{A, B}$ of $i_{A, B}^{+}$such that

$$
\mathcal{C H}_{A, B}^{+} \cap \mathcal{U}_{A, B} \subset \overline{\mathcal{T}} \text {. }
$$

We have in fact that this is true globally:

Proposition 8.1. Under the assumptions of Theorem 6 .

$$
\mathcal{C H}_{A, B}^{+} \subset \overline{\mathcal{T}} \text {. }
$$

Proof. Define $r_{0}=\min _{\Sigma} r$. We have by the Raychaudhuri equations (8), (9) that that if $(u, v) \in \mathcal{Q}$ for which $r(u, v)<r_{0}$, then $(u, v) \in \mathcal{T}$.

Now, let $\left(u_{1}, v_{1}\right) \in \mathcal{C H}_{A}^{+}$(WLOG, we shall prove the statement for $\mathcal{C H}_{A}^{+}$) for which $\left(u_{1}, v_{1}\right) \notin \overline{\mathcal{T}}$. It follows from the second statement of Theorem 4 that for each $\tilde{v}<v_{1}$ sufficiently close to $v_{1}$, there exists a $\tilde{u}(\tilde{v})$ such that $\partial_{v} r(\tilde{u}, \tilde{v})=0$, and such that $(u, \tilde{v}) \in \mathcal{T}$ for $u^{*}<u<\tilde{u}$, where $\left(u^{*}, \tilde{v}\right) \in \mathcal{A}_{A}^{\prime}$. Moreover, there exists an $\epsilon$ such that $\tilde{u} \geq u\left(\mathcal{H}^{+}\right)+\epsilon$ for all $\tilde{v}$.

Since $\partial_{v} r(\tilde{u}, \tilde{v})=0$ we have $r(\tilde{u}, \tilde{v})=\varpi \pm \sqrt{\varpi^{2}-Q^{2}}$. On the other hand, by the monotonicity and Theorem [6 above, it follows that $\varpi(\tilde{u}, \tilde{v}) \rightarrow \infty$ as $\tilde{v} \rightarrow v_{1}$. It follows that for $\tilde{v}$ sufficiently close to $v_{1}$, we have $r(\tilde{u}, \tilde{v})=\varpi-\sqrt{\varpi^{2}-Q^{2}} \rightarrow 0$ as $\tilde{v} \rightarrow v_{1}$. In particular, for $\tilde{v}$ sufficiently close to $v_{1}, r(\tilde{u}, \tilde{v})<r_{0}$ and thus $(\tilde{u}, \tilde{v}) \in \mathcal{T}$, a contradiction.

Corollary 8.1. Under the assumptions of Theorem $\square$, $\varpi$ "continuously" extends to $\infty$ on the whole of $\mathcal{C H}_{A, B}^{+}$. 
Proof. We apply Theorem [6 the above proposition and again the monotonicity (15).

As an additional corollary we have the following

Corollary 8.2. Under the assumptions of Theorem $6,(\mathcal{M}, g)$ is future inextendible as a manifold with $C^{2}$ Lorentzian metric.

Proof. Let $(\widetilde{\mathcal{M}}, \widetilde{g})$ be a non-trivial $C^{2}$ extension. We apply Part III of Proposition 4.1. Let $\gamma$ be such that $\overline{\pi_{\mathcal{Q}}\left(\left.\gamma\right|_{\mathcal{M}}\right)} \cap \mathcal{C H}^{+} \neq \emptyset$. Since $\varpi$ extends "continuously" to $\infty$ on $\mathcal{C H}^{+}$, it follows from (12) that the supremum of the Kretschmann scalar on $\left.\gamma\right|_{\mathcal{M}}$ is $\infty$, a contradiction.

The above extendibility statement can be strengthened, but such statements below $C^{2}$ need care. We will examine the consequence of the blow-up of mass in our setting in Section 13. Cf. the comments following Theorem 3 ,

Conjecture 2 notwithstanding, it is an open question whether Proposition 8.1 holds under the assumptions of only Theorem 4

\section{Cauchy stability}

We will apply a Cauchy stability argument in the following form. Let us fix a subextremal Reissner-Nordström solution with parameters $0<Q_{R N}<M_{R N}$, and an arbitrary spherically symmetric (but not necessarily admissible) Cauchy hypersurface $\Sigma$. We fix the gauge described before Theorem 4

We shall consider " $\delta$-perturbations" of the data on $\Sigma$. A $\delta$-perturbation will be such that (with respect to the above gauge), the following pointwise inequalities hold on $\Sigma$ :

$$
\begin{aligned}
\left|r-r_{R N}\right|<\delta, & & \left|\Omega-\Omega_{R N}\right|<\delta, \\
\left|\partial_{u} r-\partial_{u} r_{R N}\right|<\delta, & & \left|\partial_{v} r-\partial_{v} r_{R N}\right|<\delta, \\
\left|\partial_{u} \Omega-\partial_{u} \Omega_{R N}\right|<\delta, & & \left|\partial_{v} \Omega-\partial_{v} \Omega_{R N}\right|<\delta, \\
\left|Q-Q_{R N}\right|<\delta, & & \left|\varpi-M_{R N}\right|<\delta, \\
\left|\partial_{u} \phi\right|<\delta, & & \left|\partial_{v} \phi\right|<\delta .
\end{aligned}
$$

Note that in the two-end case, our equations admit a local well-posedness theory in $C^{1}$ of $(\Omega, r, \phi)$. Our Cauchy stability statement is then as follows:

Proposition 9.1. Given any $0<U, V<\infty$ and $\epsilon>0$ there exists a $\delta$ so that for $\delta$-perturbations of subextremal Reissner-Nordström data, the maximal Cauchy development $(\mathcal{M}, g, F, \phi)$ contains the compact null rectangle $[-U, U] \times[-V, V]$ and the estimates (23)-(27) hold there, with $\epsilon$ in place of $\delta$.

In particular, we have,

Corollary 9.1. Let $\epsilon>0$. Consider an arbitrary data set which is a $\delta$-perturbation of Reissner-Nordström data with parameters $M_{R N}, Q_{R N}$, and let $(\mathcal{M}, g, F, \phi)$ be the maximal Cauchy development. Then for $\delta$ sufficiently small there exist past and future admissible Cauchy hypersurfaces $\Sigma_{ \pm} \subset \mathcal{M}$, which coincide with $\Sigma$ for large values of $|x|$, and such that the induced data on $\Sigma_{ \pm}$are $\epsilon$-perturbations of Reissner-Nordström data on $\Sigma_{ \pm}$, with respect to the normalisation described before




In view of the above corollary, we are justified in having restricted up to now attention on the case $\Sigma=\Sigma_{+}$, and we shall always restrict our attention in what follows to the case $\Sigma=\Sigma_{+}$, and to future evolution, in the proof of Theorem 3 .

\section{A Uniform ESTimate on the Future BOUNDARY OF THE 'STABLE BLUE-SHIFT' REGION}

To prove the main theorem of the present paper, we appeal directly to another statement from [17, which is used in the proof of relation (20) of Theorem 4 .

Proposition 10.1 (see Proposition 9.1 of [17]). Under the assumptions of Theorem 4, there exists a spacelike curve $\gamma_{A}$ terminating at $i_{A}^{+}$,

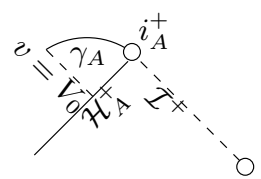

an $s>0, a C_{s}>0$, such that on $\gamma_{A}$,

$$
0<-\partial_{v} r \leq C_{s} v^{-1-s}
$$

and such that given any $\epsilon>0$, there exists a $V_{0}$ such that

$$
\left|r-\varpi_{A}^{+}+\sqrt{\left(\varpi_{A}^{+}\right)^{2}-Q^{2}}\right| \leq \epsilon
$$

on $\gamma_{A} \cap\left\{v \geq V_{0}\right\}$.

Examining the proofs of [17, 20, we easily see

Claim. Given $\epsilon_{0}>0$ and $C>0$, then there exists a $\delta_{0}>0$ such that for all $\delta_{0}$ perturbations of a fixed sub-extremal Reissner-Nordström data-set with parameters $0<Q_{R N}<M_{R N}$ on an admissible $\Sigma$, and such that moreover (18) holds with constant $C$, then (28) holds on $\gamma_{A}$ where $C_{s}, s$ can be chosen uniformly, and such that (29) holds with $\epsilon=\epsilon_{0}$ for a $V_{0}$ that can be chosen uniformly.

Note that the position of $\gamma_{A}$ in $(u, v)$ coordinates depends of course on the solution.

Finally, we remark that an identical construction gives us $\gamma_{B}$, analogous to $\gamma_{A}$, with $u$ replacing $v$.

Remark 10.1. Let us note here that $\gamma_{A}$ above represents, in the terminology introduced in [17, the future boundary of the "stable blue shift region" $\mathcal{B}_{\gamma}$. See Section 6 of [17. for a discussion of these regions. As we shall see, the significance of (28) is that it is integrable in $v$, and this property will persist to the future of $\gamma_{A}$.

In the course of the proof of our main theorem, we will not in fact appeal to Theorem 4 or Corollary 7.1, but rather directly to Proposition 10.1. In particular, we shall effectively recover the proof of the non-emptiness relation (20), given of course Proposition 10.1. 


$$
\text { 11. } \mathcal{S} \cup \mathcal{S}_{A} \cup \mathcal{S}_{B}=\emptyset
$$

Having recalled all that we will need, we may now prove the main result of the paper. Given the framework of Proposition 4.1 and the comment following Corollary 9.1, then the first two statements of Theorem 3 of the introduction will follow from

Theorem 7. Let $0<Q_{R N}<M_{R N}$. Let $C>0$ be a constant. Then there exists a $\delta>0$ such that for all $\delta$-perturbations of subextremal Reissner-Nordström data on a future admissible $\Sigma$ with parameters $Q_{R N}, M_{R N}$, satisfying moreover (18), then

$$
\mathcal{S} \cup \mathcal{S}_{A} \cup \mathcal{S}_{B}=\emptyset .
$$

Moreover, given $\epsilon>0$, then the global bound

$$
r \geq M_{R N}-\sqrt{M_{R N}^{2}-Q_{R N}^{2}}-\epsilon
$$

holds, for $\delta$ chosen small enough.

Proof. Given $\epsilon>0$ and $C$, let $0<\epsilon_{0} \ll \epsilon$ (to be determined), and let $\delta_{0}, V_{0}, s, C_{s}$ be as in the claim following Proposition 10.1 We have

$$
\left|Q-Q_{R N}\right|<\epsilon_{0} .
$$

We can then choose $V \geq \max \left\{V_{0},-v\left(\mathcal{H}_{B}^{+}\right), 0\right\}$ such that

$$
\int_{V}^{v} 2 C_{s} v^{-1-s}<\epsilon_{0}
$$

and

$$
\left|r(u, v)-\left(M_{R N}-\sqrt{M_{R N}^{2}-Q_{R N}^{2}}\right)\right|<\epsilon_{0}
$$

for $(u, v) \in \gamma_{A}$ and $v \geq V$.

We apply the same argument to the end $B$ and this gives us a $U_{0}$, a new $\delta_{0}$, and a $\gamma_{B}$. Let us call $\delta_{0}$ the minimum of the new $\delta_{0}$ and the previous. We may then choose a $\left.U \geq \max \left\{U_{0},-u\left(\mathcal{H}_{A}^{+}\right), 0\right)\right\}$ satisfying the analogue of (31) with $u, U$, in place of $v, V$.

Let us note that in the Reissner-Nordström solution with parameters $M_{R N}$, $Q_{R N}$, we have that

$$
r_{R N} \geq M_{R N}-\sqrt{M_{R N}^{2}-Q_{R N}^{2}}+\epsilon_{U V}
$$

on the rectangle $[-U, U] \times[-V, V]$, while

$$
r_{R N} \leq M_{R N}-\sqrt{M_{R N}^{2}-Q_{R N}^{2}}+\epsilon_{0}
$$

on $\left[u_{A, R N}(V), U\right] \times\{V\},\{U\} \times\left[v_{B, R N}(U), V\right]$, where by definition, $\left(u_{A, R N}(V), V\right) \in$ $\gamma_{A, R N},\left(U, v_{B, R N}(U)\right) \in \gamma_{B, R N}$.

Now given these $U, V$ and choosing $\epsilon_{1} \ll \epsilon_{U V}$, $\epsilon_{0}$, we apply Proposition 9.1 (with $\epsilon_{1}$ in place of $\epsilon$ ), and let $\delta$ be the minumum of the previous $\delta_{0}$ and that given by the above corollary.

For $\epsilon_{1} \ll \epsilon_{U V}, \epsilon_{0}$ sufficiently small, it follows that on $\left[u_{A}(V), U\right] \times\{V\}$ and $\{U\} \times\left[v_{B}(U), V\right]$, we have

$$
r \geq M_{R N}-\sqrt{M_{R N}^{2}-Q_{R N}^{2}}-\epsilon_{0}
$$


and also

$$
\begin{gathered}
\varpi>Q, \\
\varpi-\sqrt{\varpi^{2}-Q^{2}}<r<\varpi+\sqrt{\varpi^{2}-Q^{2}},
\end{gathered}
$$

and thus, $1-2 m / r<0$. In view of the fact that the signs of $\partial_{u} r, \partial_{v} r$, respectively are determined by Raychaudhuri on these sets, it follows that $\left[u_{A, R N}(V), U\right] \times\{V\} \cup$ $\{U\} \times\left[v_{B, R N}(U), V\right] \subset \mathcal{T}$. In view of this together with the first inequality of (28) and its $u$-analogue, we obtain

$$
J^{+}\left(\gamma_{A}\right) \cup J^{+}\left(\gamma_{B}\right) \cap(\{v \geq V\} \cup\{u \geq U\}) \subset \mathcal{T} .
$$

We will show that (32) holds on the region (34) with $\epsilon_{0}$ replaced by $3 \epsilon_{0}$.

For this, let us define the region

$\mathcal{X}=J^{+}\left(\gamma_{A}\right) \cup J^{+}\left(\gamma_{B}\right) \cap(\{v \geq V\} \cup\{u \geq U\}) \cap\left\{r \geq M_{R N}-\sqrt{M_{R N}^{2}-Q_{R N}^{2}}-4 \epsilon_{0}\right\}$

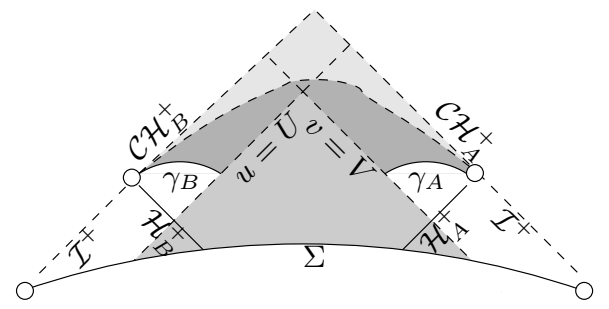

In view of (33), $\mathcal{X} \supset\left[u_{A}(V), U\right] \times\{V\} \cup\{U\} \times\left[v_{B}(U), V\right]$, while in view of (32), $\mathcal{X} \supset\left(\gamma_{A} \cap\{v \geq V\}\right) \cup\left(\gamma_{B} \cap\{u \geq U\}\right)$.

By (34), we have that $\mathcal{X} \subset \mathcal{T}$. We have thus the one-sided bound

$$
r \leq M_{R N}-\sqrt{M_{R N}^{2}-Q_{R N}^{2}}+\epsilon_{0}
$$

Let us note that by the monotonicity properties of $r$, then $\mathcal{X}$ is a past set in the topology of the left hand side of (34).

Now choosing $\epsilon$ sufficiently small, then the inequality $M_{R N}>Q_{R N}$, the definition of $\mathcal{X}$ and the bound (35) yields the one-sided bound

$$
-\frac{1}{4 r}+\frac{Q^{2}}{4 r^{3}}>0
$$

in $\mathcal{X}$, and thus by (5) we have

$$
\partial_{u} \log \left(-\partial_{v} r\right) \leq \frac{1}{r}\left(-\partial_{u} r\right)
$$

Integrating in $u$ from $\gamma_{A}(v)$, we obtain in the region $\mathcal{X} \cap\{v \geq V\}$ the bound

$$
\log \left(-\partial_{v} r\right)(u, v)<E \epsilon_{0}+\log \left(-\partial_{v} r\right)\left(u_{A}(v), v\right)
$$

where $\left(u_{A}(v), v\right) \in \gamma_{A}$, and $E$ is a constant depending on $M_{R N}, Q_{R N}$. Choosing $\epsilon_{0}$ so that $e^{E \epsilon_{0}} \leq 2$, we obtain

$$
0<-\partial_{v} r<2 C_{s} v^{-1-s} .
$$

One obtains similarly to (37),

$$
0<-\partial_{u} r<2 C_{s} u^{-1-s}
$$

in $\mathcal{X} \cap\{u \geq U\}$. 
Now integrating (37) from $\left[u_{A}, U\right] \times\{v=V\} \cup\left(\gamma_{A} \cap\{v \geq V\}\right)$ one obtains in view of (31) that

$$
r \geq M_{R N}-\sqrt{M_{R N}^{2}-Q_{R N}^{2}}-2 \epsilon_{0}
$$

in $\mathcal{X} \cap\{u \leq U\}$. It follows that

$$
\mathcal{X} \supset \cup_{v \geq V}\left[\left(u_{A}(v), U\right] \times\{v\},\right.
$$

in particular

$$
\mathcal{X} \supset\{U\} \times[V, \infty) .
$$

We may now interate (38) in $u$ from $\{U\} \times\left[v_{B}, \infty\right)$ to obtain similarly

$$
r \geq M_{R N}-\sqrt{M_{R N}^{2}-Q_{R N}^{2}}-3 \epsilon_{0}
$$

in $\mathcal{X} \cap\{u \geq U\}$. This now implies that

$$
\mathcal{X}=J^{+}\left(\gamma_{A}\right) \cup J^{+}\left(\gamma_{B}\right) \cap(\{v \geq V\} \cup\{u \geq U\})
$$

with the bound (39) holding on all of $\mathcal{Q}$. The theorem follows, choosing (in addition to the above restrictions) $3 \epsilon_{0}<\epsilon$.

\section{The continuous extension}

Let $u^{\prime}>u\left(\mathcal{H}_{A}^{+}\right), v^{\prime}>v\left(\mathcal{H}_{B}^{+}\right)$. We shall define a new set of null coordinates $\left(u^{*}, v^{*}\right)$ centred at $\left(u^{\prime}, v^{\prime}\right)$, i.e. such that $\left(u^{*}, v^{*}\right)=(0,0)$ at the old $\left(u^{\prime}, v^{\prime}\right)$, and such that with respect to the new coordinates, $\left(\Omega^{*}\right)^{2}=1$ on $u^{*}=0, v^{*}=0$. Let us however immediately drop the $*$ and refer to these new coordinates again as $(u, v)$.

Since $\partial_{v} r$ becomes negative on $v=0$ and $\partial_{u} r$ becomes negative on $u=0$, then from (8), (9) and the result of the previous section, it follows that $J^{+}\left(\mathcal{H}_{A}^{+}\right) \cup J^{+}\left(\mathcal{H}_{B}^{+}\right)$ corresponds to a finite range

$$
\left(-U^{\prime}, U^{\prime \prime}\right) \times\left(-V^{\prime}, V^{\prime \prime}\right)
$$

for some $0<U^{\prime}, U^{\prime \prime}<\infty, 0<V^{\prime}, V^{\prime \prime}<\infty$.

Proposition 12.1. Under the assumptions of Theorem 7 , then with respect to the new coordinate system defined above, $\Omega^{2}, r$ and $\phi$ extend to continuous functions on the half-closed rectangle

$$
\left(-U^{\prime}, U^{\prime \prime}\right] \times\left(-V^{\prime}, V^{\prime \prime}\right]
$$

Moreover, $\Omega, r$ and $\phi$ restricted to both $\left\{U^{\prime \prime}\right\} \times\left(-V^{\prime}, V^{\prime \prime}\right)$ and $\left(-U^{\prime}, U^{\prime \prime}\right) \times\left\{V^{\prime \prime}\right\}$ are smooth functions.

Proof. We begin with $r$. The estimate of the previous section shows that for $-U^{\prime}<$ $u<U^{\prime \prime}$

$$
\int_{v_{1}}^{v_{2}}\left(-\partial_{v} r\right)(u, v) d v \rightarrow 0
$$

as $v_{1}<v_{2}$ and $v_{1} \rightarrow V^{\prime \prime}$. On the other hand, the argument of the previous section also yields the estimate

$$
\sup _{v \geq 0}-\partial_{u} r(u, v) \leq-C \partial_{u} r\left(u, V^{\prime \prime}-\epsilon\right)
$$

for $\epsilon$ sufficiently small. Since moreover

$$
\lim _{\tilde{u} \rightarrow U^{\prime \prime}} \int_{\tilde{u}}^{U^{\prime \prime}}-\partial_{u} r\left(u, V^{\prime \prime}-\epsilon\right) d u \rightarrow 0
$$


the required statement about $r$ follows immediately, interchanging also the role of $u$ and $v$.

Note in fact the bound

$$
\int_{0}^{U^{\prime \prime}} \int_{0}^{V^{\prime \prime}}\left|\partial_{u} r \partial_{v} r\right| d u d v \leq C
$$

We turn now to $\phi$. Recall from Section 13 of [17, that, in view of the global lower bound on $r$, and the bound 8

$$
\int_{\mathcal{H}_{A}^{+}}\left|\partial_{v} \phi\right| d v \leq C, \quad \int_{\mathcal{H}_{B}^{+}}\left|\partial_{u} \phi\right| d u \leq C
$$

which follow from Theorem 5 , one can obtain global uniform bounds

$$
\int_{-V^{\prime}}^{V^{\prime \prime}} \sup _{u \geq-U^{\prime}}\left|\partial_{v} \phi\right| d v \leq C, \quad \int_{-U^{\prime}}^{U^{\prime \prime}} \sup _{v \geq-V^{\prime}}\left|\partial_{u} \phi\right| d u \leq C .
$$

Note that these bounds imply the global bound

$$
|\phi| \leq C .
$$

Integrating the wave equation (7), our estimates show that for each $v \in\left(-V^{\prime}, V^{\prime \prime}\right)$, and $-U^{\prime}<u_{0},-V^{\prime}<v_{0}$, we have

$$
\sup _{u \geq u_{0}}\left|\partial_{v} \phi(u, v)\right| \leq C\left(v, u_{0}\right)
$$

and similarly

$$
\sup _{v \geq v_{0}}\left|\partial_{u} \phi(u, v)\right| \leq C\left(u, v_{0}\right)
$$

for each $u \in\left(-U^{\prime}, U^{\prime \prime}\right)$, for functions $C\left(v, u_{0}\right), C\left(u, v_{0}\right)$ such that

$$
\int_{u_{0}}^{U^{\prime \prime}} C\left(u, v_{0}\right) d u<\infty, \quad \int_{v_{0}}^{V^{\prime \prime}} C\left(v, u_{0}\right) d v<\infty .
$$

The continuity of $\phi$ now follows.

We finally turn to $\Omega$. From (35) we see, for sufficiently small $\epsilon$, the one-sided bound in the region $\left[0, U^{\prime \prime}\right) \times\left[V^{\prime \prime}-\epsilon, V^{\prime \prime}\right) \cup\left[U^{\prime \prime}-\epsilon, U^{\prime \prime}\right) \times\left[0, V^{\prime \prime}\right)$ :

$$
\partial_{u} \partial_{v} \log \Omega^{2} \leq-4 \pi \partial_{u} \phi \partial_{v} \phi+\frac{1}{r^{2}} \partial_{v} r \partial_{u} r .
$$

From (41), it follows that the first term on the right hand side of (43) is bounded upon integration in absolute value over $\left[-U^{\prime}, U^{\prime \prime}\right] \times\left[-V^{\prime}, V^{\prime \prime}\right]$, while from (40), the second term on the right hand side of (43) is bounded upon integration in absolute value over $\left[0, U^{\prime \prime}\right] \times\left[0, V^{\prime \prime}\right]$. Thus, these terms remain bounded when integrated over subregions.

Integrating now (6) in $[0, u] \times[0, v]$, for $u \geq 0, v \geq 0$, using the compactness of $\left[0, U^{\prime \prime}-\epsilon\right] \times\left[0, V^{\prime \prime}-\epsilon\right]$, the inequality (43) in the remaining region, and the bounds of the preceding paragraph, we obtain thus the uniform one sided bound

$$
\log \Omega^{2} \leq C \text {. }
$$

In particular, we have the global bound

$$
\int_{0}^{U^{\prime \prime}} \int_{0}^{V^{\prime \prime}} \Omega^{2} d u d v<\infty
$$

\footnotetext{
${ }^{8}$ note these are independent of the normalisation of the null coordinates
} 
Now let us note that the above argument would have applied if we chose $u=0$, $v=0$ at an earlier time (always strictly to the future of the two event horizons), and (44) is a coordinate invariant statement!

It follows that for any $-U^{\prime}<u_{0}<U^{\prime \prime},-V^{\prime}<v_{0}<V^{\prime \prime}$, we have

$$
\int_{u_{0}}^{U^{\prime \prime}} \int_{v_{0}}^{V^{\prime \prime}} \Omega^{2} d u d v<C\left(u_{0}, v_{0}\right)
$$

Using (45) and again (41), we may now integrate (6) backwards and forwards from $u=0, v=0$, to obtain, in $\left[u_{0}, U^{\prime \prime}\right) \times\left[v_{0}, V^{\prime \prime}\right)$, a two-sided bound

$$
\left|\log \Omega^{2}\right| \leq C\left(u_{0}, v_{0}\right) \text {. }
$$

Revisiting the equation (6), we obtain similar bounds

$$
\begin{aligned}
& \sup _{u \geq u_{0}}\left|\partial_{v} \Omega\right|(u, v) \leq C\left(v, u_{0}\right), \\
& \sup _{v \geq v_{0}}\left|\partial_{u} \Omega\right|(u, v) \leq C\left(u, v_{0}\right),
\end{aligned}
$$

where $C\left(u, v_{0}\right), C\left(v, u_{0}\right)$ again satisfy (42).

The continuous extendibility of $\Omega$ follows.

We have in fact already proven more, namely, that, in addition to continuous extendibility, $r, \phi$ satisfy global $B V$ bounds on both $\left(-U^{\prime}, U^{\prime \prime}\right] \times\left\{V^{\prime \prime}\right\}$ and $\left\{U^{\prime \prime}\right\} \times$ $\left(-V^{\prime}, V^{\prime \prime}\right]$, while $\Omega$ satisfies local $B V$ bounds on these sets.

The higher order regularity claimed by the proposition follows inductively by differentiating the basic equations and applying the above bounds.

\section{Blow-up of Christoffel symbols}

Given the above continuous extension, let us examine in more detail the geometry of the singular surface in the case where the assumptions of Theorem 6 apply. We assume thus that in addition to the assumptions of Theorem 7 , we have (22), and consequently by Corollary $8.1 \varpi$ extends "continuously" to $\infty$ identically on $\mathrm{CH}^{+}$ (let us note that the monotonicity shows that we have "continuity" also at the bifurcation point).

With respect to the finite coordinates defined in the last section, which are to be thought now as a regular coordinate system of a $C^{1}$ (in fact $C^{\infty}$ ) manifold admitting a $C^{0}$ continuous extension $\widetilde{g}$ of $g$, we see from the definition of Hawking mass (11) and the bounds on $\Omega, \partial_{u} r$ that for $-U^{\prime}<u<U^{\prime \prime}$,

$$
\lim _{v \rightarrow V^{\prime \prime}} \partial_{v} r(u, v) \rightarrow-\infty
$$

and (in view of the bounds on $\partial_{v} r$ )

$$
\lim _{u \rightarrow U^{\prime \prime}} \partial_{u} r(u, v) \rightarrow-\infty .
$$

In particular, the extension $\widetilde{g}$ cannot have $C^{0}$ Christoffel symbols in $u, v$ coordinates (for instance, $\Gamma_{A B}^{u}=2 \Omega^{-2} r \partial_{v} r \sigma_{A B}$, with $A, B$ coordinates on $\mathbb{S}^{2}$ ).

From equations (8), (9), in view again of the bounds on $\Omega$, it follows that for $-U^{\prime}<u<U^{\prime \prime}$

$$
\int_{v}^{V^{\prime \prime}}\left(\partial_{v} \phi\right)^{2}(u, v) d v=\infty
$$


while for $-V^{\prime}<v<V^{\prime \prime}$

$$
\int_{u}^{U^{\prime \prime}}\left(\partial_{u} \phi\right)^{2}(u, v) d u=\infty
$$

It follows in particular that $\phi$ does not extend $H_{\text {loc }}^{1}$ at any point of $\mathcal{C H}^{+}$.

To continue, let us note first that we know a bit more from [17, namely that, under the assumptions of Theorem 6, then in a neighbourhood $\mathcal{U}_{A}$ of $i_{A}^{+}$, then WLOG, $\partial_{u} \phi>0, \partial_{v} \phi>0$ in $\mathcal{U}_{A} \cap J^{+}\left(\gamma_{A}\right)$, and thus, noting from (7) that it follows that $\partial_{v}\left(r \partial_{u} \phi\right)>0$, we have that $\partial_{u} \phi>0$ on $\mathcal{C H}_{A}^{+} \cap \mathcal{U}_{A}$. (Let us note moreover that this and our upper bounds on $\partial_{u} \phi$ imply that in fact $\partial_{v} \phi>0$ in a neighbourhood of $\left(-U^{\prime}, U^{\prime \prime}\right) \times\left\{V^{\prime \prime}\right\}$.)

Let $u_{1}<u_{2}$ be two $u$-values such that $u=u_{1}$ and $u=u_{2}$ intersect $\mathcal{C H}_{A}^{+} \cap \mathcal{U}_{A}$. Note by the continuity properties of $\left.\partial_{u} \phi\right|_{\mathcal{C H}_{A}^{+}}$and compactness, we have for $v_{0}<V^{\prime \prime}$ suitable large, a lower bound $\left|\partial_{u} \phi\right| \geq c\left(v_{0}, u_{1}, u_{2}\right)$ in $v \geq v_{0}, u_{1} \leq u \leq u_{2}$, for a positive $c>0$.

One sees easily that

$$
\begin{array}{r}
\int_{v_{0}}^{V^{\prime \prime}}\left(\partial_{v} \Omega\right)^{2}\left(u_{1}, v\right) d v+\int_{v_{0}}^{V^{\prime \prime}}\left(\partial_{v} r\right)^{2}\left(u_{1}, v\right) d v+\int_{v_{0}}^{V^{\prime \prime}}\left(\partial_{v} \Omega\right)^{2}\left(u_{2}, v\right) d v \\
\geq c \int_{v_{0}}^{V^{\prime \prime}}\left(\partial_{v} \phi\right)^{2}\left(u_{1}, v\right) d v-C
\end{array}
$$

from which it follows that there exists a $u_{1} \leq u_{0} \leq u_{2}$ such that for $u \neq u_{0}$, then

$$
\int_{v_{0}}^{V^{\prime \prime}}\left(\partial_{v} \Omega\right)^{2}(u, v) d v=\infty
$$

or

$$
\int_{v_{0}}^{V^{\prime \prime}}\left(\partial_{v} r\right)^{2}(u, v) d v=\infty
$$

In particular, the Christoffel symbols fail to be $L_{\text {loc }}^{2}$ at any point of $\mathcal{C H}_{A}^{+} \cap \mathcal{U}_{A}$ (recall $\Gamma_{A B}^{u}=2 \Omega^{-2} r \partial_{v} r \sigma_{A B}$ and note also $\Gamma_{v v}^{v}=\partial_{v} \log \Omega^{2}$ ).

Let us note that if (47) is true for a particular value of $u$, then it is true for all $u \in\left(-U^{\prime}, U^{\prime \prime}\right]$.

On the other hand, if (47) does not hold, then we easily see the following: Let $Z=\left\{u: \partial_{u} \phi\left(u, V^{\prime \prime}\right) \neq 0\right\}$. We have that for almost all $u \in Z$, (46) holds.

Similar considerations apply to $\left\{u=U^{\prime \prime}\right\}$.

It follows thus that the Christoffel symbols fail to be $L_{\text {loc }}^{2}$ at any point $x$ of $\mathcal{C H}^{+}$ such that $\left.\phi\right|_{\mathrm{CH}^{+}}$is not constant in a neighbourhood of $x$.

One expects that the statements shown in this section can be generalised to apply to any nontrivial $C^{0}$ Lorentzian manifold $(\widetilde{\mathcal{M}}, \widetilde{g})$ extending $(\mathcal{M}, g)$, not assumed a priori to have the above differential structure. We shall not pursue the details here.

\section{Connection With the Luk-Rodnianski theory of INTERACTing IMPULSIVE GRAVITATIONAL WAVES}

In a different guise, "weak null singularities" have a long history in general relativity. They occur in the explicit colliding plane wave spacetimes constructed by Khan-Penrose [30] and Szekeres [44, where intersecting null hypersurfaces carry 
delta-function singularites in the curvature. In these spacetimes, however, the singularities are even weaker than the "weak null singularities" of our Theorem 2 in that the Christoffel symbols remain bounded. In view of this, the plane waves of 30. were not interpreted as terminal singular boundaries of spacetime, but rather as singular hypersurfaces in an ambient spacetime continuing on both sides of the hypersurface, whose dynamics should still be uniquely prescribed by the Einstein equations, interpreted in a weak sense. I.e. the situation should be much like that for well known shock fronts of classical fluid dynamics, where the equations uniquely determine the dynamics (after imposing in addition a suitable entropy condition).

A mathematical theory incorporating the above (and more general!) singular hypersurfaces has only been achieved very recently. The reason this problem is so difficult is that this type of singularity, despite being sufficiently "weak" so as to allow for the spacetimes to be interpreted as weak solutions of the Einstein equations across the singularity, is still below the general well-posedness regularity threshold for the Einstein equations (even after the recent seminal improvement due to Klainerman-Rodnianski-Szeftel 31 requiring only that the metric be in the Sobolev space $H^{2}$ on a Cauchy surface). In remarkable very recent work of LukRodnianski 35, a well-posedness result has been achieved for the characteristic initial value problem with characteristic data admitting a delta-function singularity of the curvature across a 2 -sphere, but otherwise smooth. This singularity is then shown to propagate along a null hypersurface emanating from the 2 -sphere. These null singular hypersurfaces can be thought of as impulsive gravitational waves, generalising the plane symmetric examples of Khan-Penrose and imbedding them finally in a dynamical framework for which uniqueness is understood.

Moreover, in [36, Luk and Rodnianski show that two impulsive gravitational waves interact so that the singularity is supported in the union of two null cones. In particular, a singularity does not form before their intersection, and their "wake" is completely regular!

Although as we have said, the null singularities of Luk-Rodnianski are less singular than the weak null singularities of the present paper (as they must be to allow for a well posedness theorem for spacetimes continuing beyond them!), it is impossible not to compare the situation established in 36. with the picture of Theorem 3 . where again two weak null singularities propagate and interact (albeit now as the terminal boundary of spacetime) without a "stronger" singularity occuring first 9 The methods introduced by [35, 36] thus give hope that vacuum spacetimes with singular structure similar to that of Theorem 3 (and without underlying symmetries) will soon be constructed, parameterised by free initial data. This will be an important first step toward understanding the validity of Conjecture 1

\footnotetext{
${ }^{9}$ Moreover, one can strengthen the connection with the following remark. Were one to drop the "constraint" equations (8)-(9) and solve only the "evolution" equations (5)-(7), then one may still reasonably interpret the evolution equations alone in a generalised sense across $\mathrm{CH}^{+}$, and extend the spacetime so as to satisfy these. The hypersurfaces $\mathcal{C H}_{A, B}^{+}$can then be thought of precisely as interacting singularities in such a spacetime, which again would effectively pass through one another. It is interesting that it is specifically the impossibility of satisfying the constraint equations that exclude such extensions from being thought of as weak solutions of the Einstein equations. In this sense, the constraint equations can be thought to play a role analogous to that of an entropy condition in classical continuum physics.
} 


\section{Epilogue: Cosmological spacetimes without SPACELiKe SINGULARITIES}

As discussed before, the original expectation of "BKL"-type behaviour inside black hole regions starts from the observation that locally the interior of a Schwarzschild black hole can be viewed as a cosmological region. This relation goes both ways, however. In particular, perturbing the Reissner-Nordström-de Sitter solution, similar considerations to Reissner-Nordström apply. In fact, as we shall see, the situation is even more interesting, and the conclusions for strong cosmic censorship potentially ominous.

15.1. A rough stability result. Let us first state a basic stability result, an exact analogue of the stability part of Theorem 3. which can be proven following the methods of this paper and [17, 20, 19, 21, depends on a relatively rough understanding for the decay properties of the scalar field between the event and cosmological horizons, and holds for all subextremal values of the Reissner-Nordström-de Sitter solution.

Theorem 8. For sufficiently small spherically symmetric perturbations of subextremal Reissner-Nordström-de Sitter initial data (with topology $\mathbb{S}^{1} \times \mathbb{S}^{2}$ and $n$ black hole regions), the maximal future (past) Cauchy development has Penrose diagramme given by:

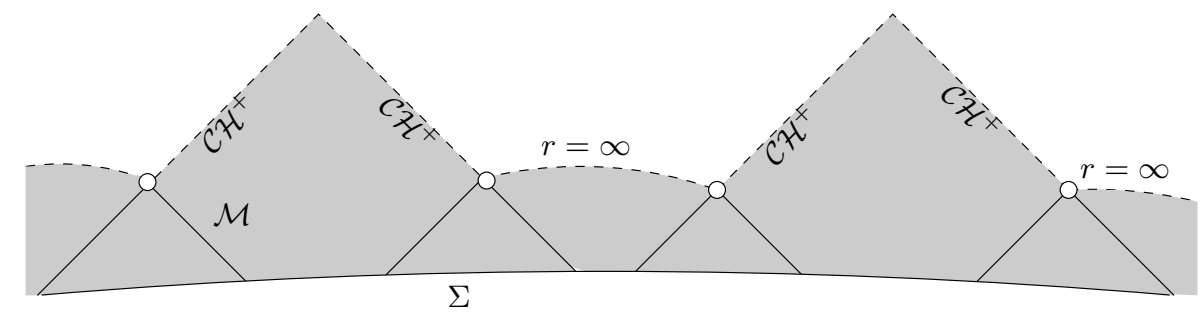

and the spacetime is extendible as a manifold with $C^{0}$-Lorentzian metric (to which $\phi$ also extends continuously), such that each connected component of $\mathrm{CH}^{+}$is a bifurcate null cone in the extension.

The above already motivates a conjecture which is stated here in the Kerr case so as to apply in fact for vacuum:

Conjecture 3. Let $(\mathcal{M}, g)$ be the maximal Cauchy development (for the Einstein vacuum equations with positive cosmological constant) of sufficiently small perturbations of subextremal Kerr-de Sitter data (with $n$ black hole regions) and initial topology $\mathbb{S}^{1} \times \mathbb{S}^{2}$. Then $(\mathcal{M}, g)$ is future (past) extendible to a $(\widetilde{\mathcal{M}}, \widetilde{g})$ with $C^{0}$ metric $\widetilde{g}$, such that $\partial \mathcal{M}$ is the union of $n$ bifurcate null cones, and all incomplete, future(past)-intextendible geodesics of $\mathcal{M}$ pass into $\widetilde{\mathcal{M}} \backslash \mathcal{M}$.

According to this conjecture, there is an open set in the moduli space of vacuum, "cosmological" spacetimes whose singularities, whether past or future, are nowhere spacelike.

15.2. The structure of the null singularity and the near extremal case. The question of the precise generic singular nature of the null boundary of these spacetimes is more delicate and depends on the parameters of the Reissner-Nordströmde Sitter spacetime which is initially perturbed. 
This problem was plagued by much confusion and several incorrect claims. The original studies of the charged spherical toy models gave a detailed analysis of the expected singular behaviour, identifying a parameter range (i) where the mass inflation scenario holds just as in the asymptotically flat case, a parameter range (ii) where the curvature blows up without the mass blowing up, and, finally, and most surprisingly, a parameter range (iii) where the curvature remains continuous. See in particular 5, 6] and the survey [10. These original studies [5, 6, however, had failed to properly address the effect of backscattering in the region between the cosmological and event horizons, already present in the scalar field model, which heuristically generates an exponentially decaying tail with the slower exponential rate governed by the surface gravity of the event horizon, as opposed to the faster rate governed by the surface gravity of the cosmological horizon. When this was taken into account, the range of stability (iii) predicted in [5, 6] disappears. See [8]. This suggested that the " $C^{2}$-inextendibility formulation" of strong cosmic censorship holds in the case of positive cosmological constant-hence the reassuring title of 8 .

Subsequent insights [14 into the proper formulation of strong cosmic censorship coming from the PDE point of view, coupled with the recent Luk-Rodnianski theory 35 described in the previous section, suggest that- 8 not withstandingone should revisit the more detailed previous analyses of the studies described just above. A revised heuristic analysis in the spirit of 6 but correctly taking into account the behaviour of the scalar field outside the event horizon 8 suggests that there is still a qualitative difference in the nature of the blow-up, depending on the parameters, corresponding to (i) and (ii) above. In particular, close to extremality, this suggests that although the curvature blows up, this is not associated with mass blow up, and, particularly relevant to the point of view advanced here, the metric in fact extends Lipschitz (its Christoffel symbols are uniformly bounded), and the scalar field extends in $H_{\mathrm{loc}}^{1}$.

Conjecture 4. In Theorem 8, if the initial data is sufficiently close to a ReissnerNordström-de Sitter with parameters in turn sufficiently close to extremal (but still subextremal!), then the spacetime extensions $(\widetilde{\mathcal{M}}, \widetilde{g})$ can be chosen so that $\widetilde{g}$ is Lipschitz, and such that the scalar field extends to $\widetilde{\mathcal{M}}$ as an $H_{\text {loc }}^{1}$ function.

For generic such initial data (for all sub-extremal values of the Reissner-Nordströmde Sitter reference solution), $\widetilde{g}$ cannot be $C^{2}$ and $\phi$ cannot extend $C^{1}$.

In fact, the linearised analysis suggests that the scalar field will extend in $W^{1, p}$, where one can take $p \rightarrow \infty$ as the initial data approaches extremality. It is slightly amusing that the closer the black hole is to extremality, the more "stable" its Cauchy horizon, when set against the backdrop of the novel instability discovered by Aretakis [1, 22 in his study of the wave equation on exactly extreme Reissner-Nordström; this then suggests that, as the parameters of the initial reference Reissner-Nordström-de Sitter are pushed towards extremality, the size of the allowed "open set" in the moduli space of data, corresponding to the domain of stability, must be taken to shrink.

When "translated" to the vacuum case (see the comments at the end of Section 3) one may expect that the $C^{1}$ blow up of the scalar field will be reflected as a $C^{0}$ blow up of the Christoffel symbols, and thus, the metric will not be Lipschitz. On the other hand, the above still suggests that the Christoffel symbols will be 
in $L_{\text {loc }}^{2}$ (in fact, in any $L_{\text {loc }}^{p}$, given data sufficiently close to extremality). This would represent a type of singularity which, although outside the general $H^{2}$-well posedness theory of [31, may be describable by a suitable generalisation of LukRodnianski theory [35] outlined in the previous section, and thus such solutions may in fact admit a local uniqueness characterization. Thus, one is led to:

Conjecture 5. Let $(\mathcal{M}, g)$ be the maximal Cauchy development of sufficiently small perturbations of Kerr-de Sitter data (with n black hole regions) and initial topology $\mathbb{S}^{1} \times \mathbb{S}^{2}$ with parameters sufficiently close to extremality. Then one can choose extensions $(\widetilde{\mathcal{M}}, \widetilde{g})$ as above such that $\widetilde{g}$ has locally square integrable Christoffel symbols and such that moreover the extensions satisfy the Einstein equations in a generalised sense. In particular, the "inextendible as a metric with $L_{\text {loc }}^{2}$ Christoffel symbols" formulation (see [14) of strong cosmic censorship does not hold in the case of positive cosmological constant.

From a sufficiently coarse point of view, the spacetimes $(\mathcal{M}, g)$ described by the above conjecture would be stable examples of cosmological black hole spacetimes without singularities-full stop.

15.3. Is strong cosmic censorship false? It may be tempting, in view of Conjecture 5. to prefer the $C^{2}$ formulation of strong cosmic censorship, and to invoke the pointwise blow-up of curvature as an indication that the effects of quantum gravity will somehow save the day. Until that time where such considerations can be well formulated, however, the local PDE-properties governing the Cauchy problem for the Einstein equations provide the best insight into the theory that we have, and its conclusions-like them or not-cannot be taken lightly. Thus, these considerations suggest that while the uniqueness property captured by strong cosmic censorship may indeed hold in the realm of gravitational collapse, its validity as a fundamental physical principle now appears in doubt.

\section{ACKNOWLEDGEMENTs}

This paper was inspired by a conversation with Amos Ori at the "Numerical relativity beyond astrophysics workshop" (hosted by the International Centre for Mathematical Sciences, Edinburgh, July 2011), whom the author also thanks for his subsequent input which has led to several improvements, particularly with regard to the discussion of the cosmological case. The author thanks the organisers of the workshop (David Garfinkle, Carsten Gundlach and Luis Lehner) for facilitating a stimulating discussion session on null singularities. Additional thanks go to Igor Rodnianski, Stefanos Aretakis, as well as Jonathans Kommemi and Luk. The author is supported in part by a grant from the European Research Council.

\section{REFERENCES}

[1] S. Aretakis Stability and instability of extreme Reissner-Nordström black hole spacetimes for linear scalar perturbations I Comm. Math. Phys. 307 (2011), 17-63

[2] S. Aretakis Stability and instability of extreme Reissner-Nordström black hole spacetimes for linear scalar perturbations II Ann. Henri Poincaré 8 (2011), 1491-1538

[3] V. A. Belinskii, I. M. Khalatnikov, and E. M. Lifshitz Oscillatory approach to a singular point in the relativistic cosmology Adv. Phys. 19, 525 (1970)

[4] A. Bonanno, S. Droz, W. Israel and S. M. Morsink Structure of the charged spherical black hole interior Proc. R. Soc. Lond. A 450 (1995), 553-567 
[5] P. R. Brady and E. Poisson Cauchy horizon instability for Reissner-Nordstrom black holes in de Sitter space Class. Quantum Grav. 9 (1992), 121-125

[6] P. R. Brady, D. Núñez and S. Sinha Cauchy horizon singularity without mass inflation Phys. Rev. D 47 (1993), 4239-4243

[7] P. Brady and J. D. Smith Black hole singularities: a numerical approach Phys. Rev. Lett. 75 (1995), no. 7, 1256-1259

[8] P. R. Brady, I. G. Moss and R. C. Myers Cosmic Censorship: As Strong As Ever Phys. Rev. Lett. 80 (1998), 3432-3425

[9] L. M. Burko Structure of the black hole's Cauchy-horizon singularity Phys. Rev. Lett. 79 (1997), no. 25, 4958-4961

[10] C. M. Chambers The Cauchy horizon in black hole-de Sitter spacetimes Ann. Israel Phys. Soc. 13 (1997), 33-84, arXiv:gr-qc/9709025

[11] Y. Choquét-Bruhat and R. Geroch Global aspects of the Cauchy problem in general relativity Commun. Math. Phys. 14 (1969), 329-335

[12] D. Christodoulou The instability of naked singularities in the gravitational collapse of a scalar field Ann. of Math. 149 (1999), no. 1, 183-217

[13] D. Christodoulou On the global initial value problem and the issue of singularities Class. Quantum Gravity 16 (1999) A23-A35

[14] D. Christodoulou The formation of black holes in general relativity. EMS Monographs in Mathematics. European Mathematical Society (EMS), Zürich, 2009.

[15] M. Dafermos Stability and instability of the Cauchy horizon for the spherically-symmetric Einstein-Maxwell-scalar field equations, Ann. Math. 158 (2003), 875-928

[16] M. Dafermos Price's law, mass inflation, and strong cosmic censorship, in Racz, I., ed. Relativity today: Proceedings of the Seventh Hungarian Relativity Workshop, Budapeset, 2004, pp. 79-90, arXiv:gr-qc/0401121

[17] M. Dafermos The interior of charged black holes and the problem of uniqueness in general relativity Comm. Pure Appl. Math. 58 (2005), 445-504

[18] M. Dafermos Spherically symmetric spacetimes with a trapped surface Class. Quantum Grav. 22 (2005), no. 11, 2221-2232

[19] M. Dafermos and I. Rodnianski A note on boundary value problems for black hole evolutions, arXiv:gr-qc/0403034

[20] M. Dafermos and I. Rodnianski A proof of Price's law for the collapse of a self-gravitating scalar field, Invent. Math. 162 (2005), 381-457

[21] M. Dafermos and A. Rendall Strong cosmic censorship for surface-symmetric cosmological spacetimes with collisionless matter, arXiv:gr-qc/0701034

[22] M. Dafermos and I. Rodnianski The black hole stability problem for linear scalar perturbations, arXiv:1010.5137

[23] G. F. R. Ellis and A. R. King Was the big bang a whimper? Commun. Math. Phys. 39 (1974), 119-156

[24] M. L. Gnedin and N. Y. Gnedin Destruction of the Cauchy horizon in the ReissnerNordström black hole Class. Quantum Grav. 10 (1993), 1083-1102

[25] C. Gundlach, R. Price and J. Pullin Late-time behavior of stellar collapse and explosition. II: Nonlinear evolution, Phys. Rev. D 49 (1994), 890-899.

[26] R. Herman and W. A. Hiscock Strength of the mass inflation singularity, Phys. Rev. D 46 (1992), 1863-1865

[27] W. A. Hiscock Evolution of the interior of a charged black hole, Phys. Lett. 83A (1981), $110-112$

[28] S. Hod and T. Piran Mass inflation in dynamic gravitational collapse of a charged scalar field Phys. Rev. Lett. 81 (1998), 1554-1557

[29] W. Israel Descent into the maelstrom: the black hole interior, in: The black Hole, 25 years after, ed. C. Teitelboim, J. Zanelli, World Scientific, London, 1998

[30] K. A. Khan and R. Penrose Scattering of two impulsive gravitational plane waves, Nature 229 (1971), 185-186

[31] S. Klainerman, I. Rodnianski and J. Szeftel The Bounded L2 Curvature Conjecture, arXiv: 1204.1767

[32] J. Kommemi The global structure of spherically symmetric charged scalar field spacetimes, Comm. Math. Phys., Online first, arXiv:1107.0949 
[33] J. Kommemi On Penrose inequalities for spherically symmetric charged black holes, preprint, 2011

[34] J. Kommemi On Cauchy horizon stability for spherically symmetric Einstein-Maxwell-KleinGordon black holes, preprint, 2011

[35] J. Luk and I. Rodnianski Local propagation of impulsive gravitational waves, arXiv:1209.1130

[36] J. Luk and I. Rodnianski Nonlinear interactions of impulsive gravitational waves for the vacuum Einstein equations, arXiv:1301.1072

[37] A. Ori Inner structure of a charged black hole: an exact mass-inflation solution Phys. Rev. Lett. 67 (1991), 789-792

[38] A. Ori Perturbative approach to the inner structure of a rotating black hole Gen. Relativity Gravitation 29 (1997), no. 7, 881-929

[39] R. Penrose Gravitational collapse and space-time singularities Phys. Rev. Lett. 14, 57-59

[40] E. Poisson and W. Israel Inner-horizon instability and mass inflation in black holes Phys. Rev. Lett. 63 (1989), no. 16, 1663-1666

[41] E. Poisson and W. Israel Internal structure of black holes Phys. Rev. D (3) 41 (1990), no. 6, 1796-1809

[42] J. M. M. Senovilla On the boundary of the region containing trapped surfaces AIP Conf. Proc. 1122 (2009), 72-87

[43] M. Simpson and R. Penrose Internal instability in a Reissner-Nordström black hole. Internat. J. Theoret. Phys. 7 (1973), no. 3, 183-197

[44] P. Szekeres Colliding plane gravitational waves, J. Math. Phys. 13 (1972), 286-294

[45] F. Tipler Singularities in conformally flat spacetimes Phys. Lett. 64A (1977), 8-10

[46] C. Williams Asymptotic behavior of spherically symmetric marginally trapped tubes. Ann. Henri Poincaré 9 (2008), 1029-1067 